Review Article

\title{
Efficacy of school-based interventions aimed at decreasing sugar-sweetened beverage consumption among adolescents: a systematic review
}

\author{
Lydi-Anne Vézina-Im ${ }^{1,2, *}$, Dominique Beaulieu ${ }^{3,4}$, Ariane Bélanger-Gravel ${ }^{5,6}$,
} Danielle Boucher ${ }^{3}$, Caroline Sirois ${ }^{3,4}$, Marylène Dugas ${ }^{7}$ and Véronique Provencher ${ }^{1,2}$ ${ }^{1}$ School of Nutrition, Laval University, Quebec City, Quebec, Canada, GIV OA6: ${ }^{2}$ Institute of Nutrition and Functional Foods (INAF), Laval University, Quebec City, Quebec, Canada: ${ }^{3}$ Department of Nursing Sciences, Université du Québec à Rimouski (UQAR), Levis, Quebec, Canada: ${ }^{4}$ Population Health and Optimal Health Practices, CHU de Quebec Research Centre, Quebec City, Quebec, Canada: ${ }^{5}$ Department of Information and Communication, Laval University, Quebec City, Quebec, Canada: ' $Q u e b e c$ Heart and Lung Institute, Quebec City, Quebec, Canada: ${ }^{7} \mathrm{CHU}$ de Quebec Research Centre, Quebec City, Quebec, Canada

Submitted 2 September 2016: Final revision received 5 January 2017: Accepted 10 January 2017: First published online 8 February 2017

\begin{abstract}
Objective: To verify the efficacy of school-based interventions aimed at reducing sugar-sweetened beverage (SSB) consumption among adolescents in order to develop or improve public health interventions.

Design: Systematic review of interventions targeting adolescents and/or the school environment.

Setting: The following databases were investigated: MEDLINE/PubMed, PsycINFO, CINAHL and EMBASE. Proquest Dissertations and Theses was also investigated for unpublished trials.

Subjects: Adolescents were defined as individuals between the ages of 12 and 17 years.

Results: A total of thirty-six studies detailing thirty-six different interventions tested among independent samples ( $n 152$ 001) were included in the review. Twenty interventions were classified as educational/behavioural and ten were classified as legislative/environmental interventions. Only six interventions targeted both individuals and their environment. Over $70 \%$ of all interventions, regardless of whether they targeted individuals, their environment or both, were effective in decreasing SSB consumption. Legislative/environmental studies had the highest success rate (90.0\%). Educational/behavioural interventions only and interventions that combined educational/behavioural and legislative/environmental approaches were almost equally effective in reducing SSB consumption with success rates of 65.0 and $66.7 \%$, respectively. Among the interventions that had an educational/behavioural component, $61.5 \%$ were theory-based. The behaviour change techniques most frequently used in interventions were providing information about the health consequences of performing the behaviour $(72 \cdot 2 \%)$, restructuring the physical environment ( $47 \cdot 2 \%)$, behavioural goal setting (36.1\%), self-monitoring of behaviour $(33 \cdot 3 \%)$, threat to health $(30 \cdot 6 \%)$ and providing general social support (30.6\%).

Conclusions: School-based interventions show promising results to reduce SSB consumption among adolescents. A number of recommendations are made to improve future studies.
\end{abstract}

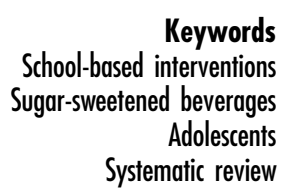

Obesity and overweight among children and youth is an important public health issue ${ }^{(1)}$. In Europe, between 9.4 and $26.4 \%$ of boys and between 6.4 and $15.9 \%$ of girls aged $10-15$ years are overweight or obese ${ }^{(2)}$. In the USA, among adolescents aged 12-19 years, 20.5\% are obese and obesity rates are slightly higher among females $(21 \cdot 0 \%)$ compared with males $(20 \cdot 1 \%)^{(3)}$. In Canada, $30 \cdot 1 \%$ of adolescents aged $12-17$ years are overweight or obese $^{(4)}$, which predisposes them to future chronic diseases $^{(5)}$. To address the obesity problem, the WHO 
recently issued new recommendations encouraging populations of all ages to limit sugar intake, including sugar-sweetened beverages (SSB), to no more than $10 \%$ and possibly $5 \%$ of total energy intake ${ }^{(6)}$.

SSB consumption contributes to excessive intake of sugar among adolescents, which is related to many health problems such as heart diseases ${ }^{(7-10)}$, stroke $^{(7)}$, obesity $^{(11-16)}$, type 2 diabetes $^{(17-20)}$, hypercholesterolaemia $^{(21,22)}$, cancer $^{(23)}$ and tooth decay ${ }^{(24)}$. Moreover, SSB offer no health benefits, increase total energy intake and may reduce the consumption of foods containing essential nutrients for optimal health, such as milk ${ }^{(6,25)}$. Unfortunately, adolescents are large consumers of SSB. $\mathrm{SBB}$ are the main source of energy from all beverages in adolescents aged 13-18 years in London, $\mathrm{UK}^{(26)}$. They are also the main sources of added sugar in Mexico, representing $66 \cdot 2 \%$ of added sugars for adolescents from 12 to 19 years of age ${ }^{(27)}$. In the USA, adolescents aged $13-18$ years drink an average of $606 \mathrm{ml}$ of soda and fruit drinks daily ${ }^{(28)}$. In Canada, boys aged 14-18 years drink a mean quantity of $574 \mathrm{~g}$ (which equates approximately the same in millilitres) of SSB daily and girls $354 \mathrm{~g}$ daily ${ }^{(29)}$.

Since habits developed during adolescence tend to be preserved throughout life ${ }^{(1)}$, it is essential to promote healthy behaviours among this population in a growing search for autonomy, especially in their food and drink choices ${ }^{(30,31)}$. Although the family environment is largely responsible for the development of healthy habits among children and youth, the responsibility of the school environment should not be underestimated given the time spent at school ${ }^{(32,33)}$. In fact, school is the ideal setting to develop and promote healthy eating habits among children and adolescents ${ }^{(32,34-36)}$. Additionally, schools offer the opportunity to easily reach young people, regardless of their age, socio-economic status (SES), cultural background and ethnicity ${ }^{(32,37,38)}$.

In the field of public health, ecological models are commonly used to design interventions aimed at changing health behaviours ${ }^{(39)}$, such as decreasing SSB consumption among adolescents. One characteristic of these models is that they recommend targeting both individuals and their environment to increase the chances of successfully changing health behaviours ${ }^{(39)}$. For example, an intervention could target adolescents by giving them information on the negative health consequences associated with consuming SSB and also target their environment by removing SSB from the vending machines and the cafeteria at their school.

Some authors recommend using theory to develop interventions that have a greater chance of changing health behaviours ${ }^{(40-45)}$. The theories most commonly used to develop public health interventions originate from social psychology (i.e. psychosocial theories) and include the Social Cognitive Theory (SCT) ${ }^{(46)}$ and its predecessor by the same author, the Social Learning Theory (SLT) ${ }^{(47)}$, the Theory of Planned Behaviour (TPB) ${ }^{(48)}$, the Transtheoretical Model (TTM) ${ }^{(49)}$ and the Self-Determination
Theory (SDT) ${ }^{(50)}$. Explaining each of these theories is beyond the scope of the present review. However, each theory gives indications on what needs to be changed in order to get individuals to intend or be motivated to change their behaviour. For example, the SCT/SLT both suggest that changing people's perception of their ability to change their behaviour - a notion known as self-efficacy - is one way to get them to change their behaviour. One advantage of the use of theory in designing public health interventions is that it can guide which techniques should be used to get participants to change their behaviour ${ }^{(51,52)}$. For example, the taxonomy of Cane et al. ${ }^{(53)}$ contains eighty-seven different behaviour change techniques originating from diverse theories that can be used to change health behaviours. There is also recent work that aims to link these behaviour change techniques to their own mechanisms of action to facilitate the development and evaluation of behaviour change interventions ${ }^{(54)}$.

In order to develop or improve school-based interventions aimed at decreasing SSB intake among adolescents, it is essential beforehand to review the scientific literature to identify which interventions and behaviour change techniques are effective at promoting this behaviour among this population. A number of reviews on various topics related to SSB and children or adolescents have already been conducted. The majority of previous reviews have focused on associations between SSB consumption and adverse health effects among children and adolescents ${ }^{(55)}$, such as increased body weight ${ }^{(56-59)}$, and also on the methodological qualities of those reviews ${ }^{(60,61)}$. One study reviewed methods to assess intake of SSB in adults, adolescents and children ${ }^{(62)}$. A few studies have reviewed the impact of policies $^{(63)}$ and additional taxes ${ }^{(64,65)}$ on children's and adolescents' consumption of SSB. Finally, there are a number of published reviews on interventions to reduce SSB consumption in children and adolescents ${ }^{(66,67)}$, including school-based interventions ${ }^{(68)}$, or to prevent childhood and adolescent obesity ${ }^{(69)}$. However, those existing reviews did not specifically target adolescents, and also included children $^{(66-69)}$, and none of them assessed the behaviour change techniques used to decrease SSB consumption.

The aim of the present study was to fill this gap in the literature by performing a systematic review of school-based interventions aimed at reducing SSB consumption among adolescents aged 12-17 years. A second objective was to identify the behaviour change techniques most effective at decreasing SSB consumption using the taxonomy of Cane et $a l .{ }^{(53)}$ in order to inform future school-based interventions aimed at changing this behaviour among adolescents.

\section{Methods}

The study protocol was registered in PROSPERO (https:// www.crd.york.ac.uk/PROSPERO/) by Do.B. in May 2015 (no. 42015023582). 


\section{Study eligibility criteria}

Population

Adolescents were defined as individuals between the ages of 12 and 17 years. Studies including participants aged $<12$ years or $>17$ years were included only if $\geq 80 \%$ of the participants were individuals between the ages of 12 and 17 years or if the mean age was between these ages.

\section{Intervention}

To be considered school-based, interventions had to be carried out in a school setting or the authors had to refer to their intervention as school-based. Studies evaluating the impact of school nutrition policies were included. Community-based interventions or those carried out outside schools were not included in the review.

\section{Outcome}

Articles had to report information on individual SSB consumption to be included in the review. There were no criteria on how individual consumption of SSB needed to be reported in the articles (e.g. millilitres or number of glasses per day or per week, percentage of individuals who reported consuming a given quantity of SSB, etc.). SBB included regular (non-diet) soft drinks, fruit drinks (excluding 100\% pure fruit juices), energy drinks, sports drinks, sweetened tea and coffee (iced or hot) and other beverages with added sugar (e.g. slush) ${ }^{(70)}$. There were also no criteria on how studies that included multiple types of beverages in their SSB definition needed to report this outcome; it could be reported separately (e.g. soft drinks, fruit drinks, etc.) or collectively (i.e. for all SSB). Studies whose definition of SSB included $100 \%$ pure fruit juices were not included in the review, except if this information was presented separately from other SBB. When unsure about whether or not SBB included $100 \%$ pure fruit juices, the authors of the articles were personally contacted by Do.B. Studies reporting information on SSB availability or SSB sales in schools were not included, since they are not measures of individual consumption of SSB.

\section{Study designs}

Types of study design included were randomised controlled trials (RCT), quasi-experimental studies and one-group pre-post studies.

\section{Exclusion criteria}

Articles written in languages other than English or French were excluded. Qualitative studies were also excluded given that the objective was to perform a meta-analysis of the results of interventions.

\section{Search strategy}

The following databases were investigated: MEDLINE/ PubMed (1950+), PsycINFO (1806+), CINAHL (1982+) and EMBASE (1974+). Proquest Dissertations and Theses
(1861+) was also investigated for grey literature (i.e. unpublished trials). There was no restriction on the year of publication of the articles. The search was performed by L.-A.V.-I. on 2 July 2015 and was updated by the same author on 21 December 2016 to include articles published until 1 December 2016. In each database, the search strategy included terms related to three major themes: SSB, adolescents and school interventions (see the online supplementary material, Supplemental File 1, for the complete search strategy). The search was developed with an experienced librarian. Additional studies were included by checking the references of the articles included in the systematic review (i.e. secondary references).

\section{Study selection and data extraction}

All articles were first screened by L.-A.V.-I. for possible duplicates and then according to their title and abstract (see Fig. 1). Clearly irrelevant articles were excluded at this step. The remaining articles were fully retrieved (full text) and two authors (L.-A.V.-I. and Do.B.) independently assessed them for eligibility. A few studies reported results based on the same sample and/or the same intervention. To avoid duplication of results and attributing more weight to these studies, only the study that had the best methodological qualities (e.g. RCT with bigger sample size $v$. one-group pre-post pilot study) and that reported the most information (e.g. baseline, post-test and follow-up data $v$. baseline and post-test data only) was included for further analysis.

Data were extracted independently by two reviewers (L.-A.V.-I. and Do.B., A.B.-G., Da.B., C.S. or M.D.*) using a standardised data extraction form. Data extracted included information on the study population, intervention, types of SSB included in the study and their measure, use of theory, behaviour change techniques used and results of the intervention. Interventions were classified as educational/ behavioural and/or legislative/environmental depending on whether they targeted individuals (e.g. nutritional education on SSB) or their environment (e.g. ban on SSB in schools) or both. The quality of studies was assessed using the Quality Assessment Tool for Quantitative Study of the Effective Public Health Practice Project (EPHPP) ${ }^{(71)}$. This tool, recommended by the Cochrane Collaboration $^{(72)}$, was selected because it can be used for different kinds of quantitative study design (RCT, one-group pre-post, etc.) and because it is especially formulated for public health studies. Briefly, the EPHPP tool evaluates the quality of studies using the following six criteria: (i) selection bias; (ii) study design; (iii) confounders; (iv) blinding; (v) data collection method; and (vi) withdrawals and dropouts. The rating for each of the six components is used to obtain a global rating of a study's

* L.-A.V.-I. extracted data (i.e. first data extraction) for all of the articles included in the review. Do.B., A.B.-G., Da.B., C.S. and M.D. shared the responsibility of performing the second data extraction and thus each extracted the data for a selection of articles. 


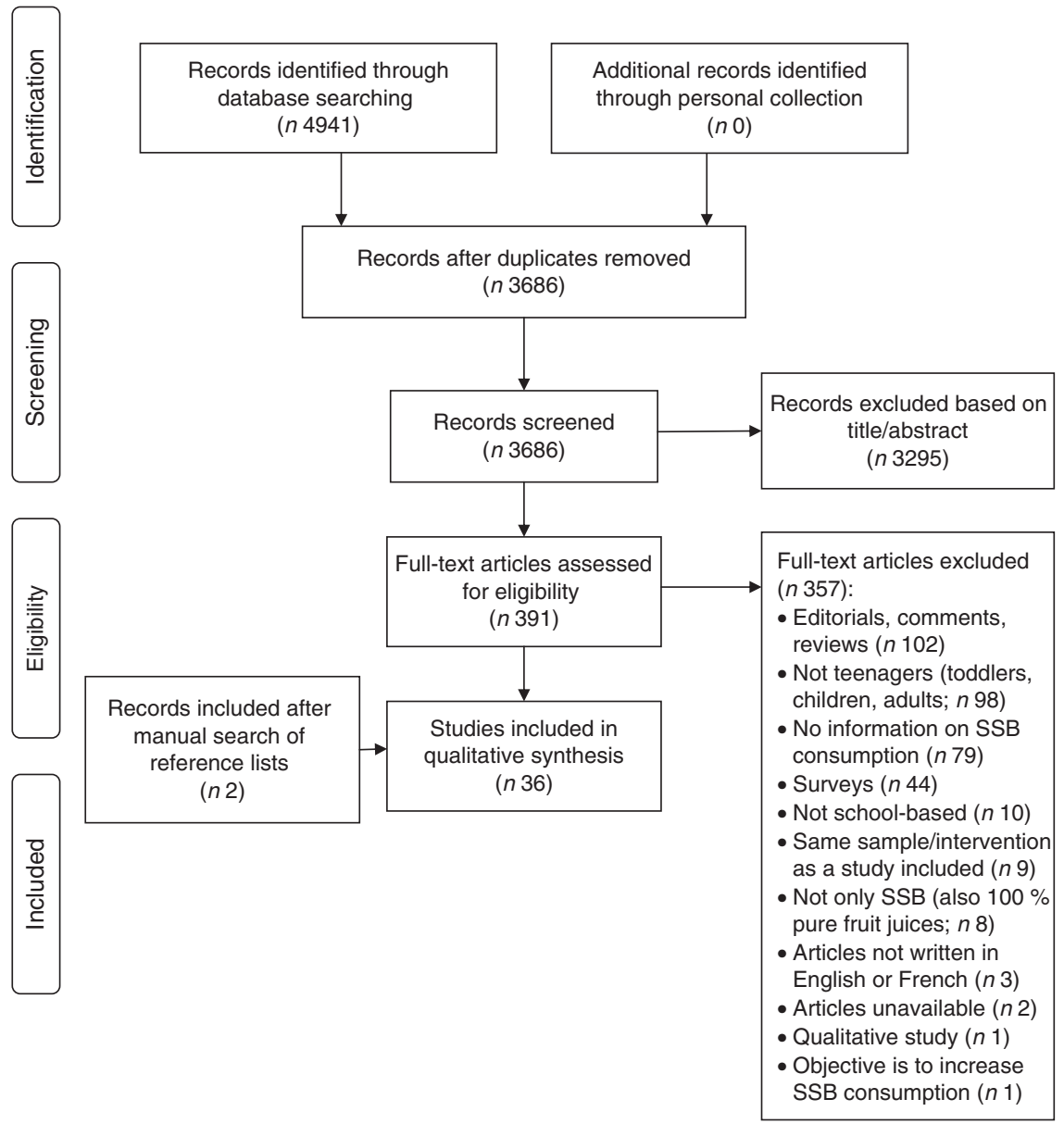

Fig. 1 PRISMA (Preferred Reporting Items for Systematic Reviews and Meta-Analyses) flowchart ${ }^{(134)}$ showing selection of the studies included in the present systematic review (SSB, sugar-sweetened beverage)

quality. A strong global rating is obtained when there are no weak ratings to any of the six components of the EPHPP. A moderate global rating is obtained when there is one weak rating and a weak global rating when there are two or more weak ratings ${ }^{(71)}$. Behaviour change techniques used in interventions were classified according to the taxonomy of Cane et al. ${ }^{(53)}$ which contains eightyseven different behaviour change techniques (e.g. restructuring the physical environment, behavioural goal setting, self-monitoring of behaviour, etc; see online supplementary material, Supplemental File 2, for the complete list of behaviour change techniques). Disagreements at each step were resolved by discussion and when no consensus could be reached a third reviewer (Do.B. or A.B.-G., depending on who originally performed the second data extraction) helped resolve the discrepancy.

\section{Results}

The results of the search strategy and its update are presented in Fig. 1. A total of thirty-six studies detailing thirty-six independent interventions were included in the present systematic review (see Table 1). This represented a total of 152001 participants at baseline. Seventy-five per cent of studies were conducted in North America (USA: twenty-four studies ${ }^{(38,73-95)}$; Canada: three stu$\left.\operatorname{dies}^{(96-98)}\right)$. Two studies were conducted in Australia ${ }^{(99,100)}$ and another two in Belgium ${ }^{(101,102)}$. Finally, one study was conducted in each of the following countries: Brazil ${ }^{(103)}$, China $^{(104)}$, India ${ }^{(105)}$, Korea ${ }^{(106)}$ and The Netherlands ${ }^{(107)}$. Given the important heterogeneity observed between the studies (i.e. differences in populations, study designs, types of intervention, behaviour change techniques used, behavioural measures and type of SSB included), no metaanalyses of the results were performed. In the rest of the text, the letter $k$ will be used to represent the number of studies and the letter $n$ to represent the number of participants.

\section{Characteristics of interventions}

Close to $60 \%$ of interventions $(58.3 \%, k 21)$ included were aimed at reducing SSB consumption as part of a general objective: the promotion of healthy eating and physical activity combined $(k 17)^{(38,76-78,83,85,87,89,91,92,97,100-103,105,107)}$ 
Table 1 Summary of the studies included in the present systematic review

\begin{tabular}{|c|c|c|c|c|c|c|c|c|}
\hline $\begin{array}{l}\text { Authors, year, } \\
\text { reference \& country }\end{array}$ & $\begin{array}{l}\text { Objective of the study or } \\
\text { intervention }\end{array}$ & Population & $\begin{array}{l}\text { Study design \& } \\
\text { quality rating* }\end{array}$ & $\begin{array}{l}\text { Baseline characteristics } \\
\text { of sample }\end{array}$ & $\begin{array}{l}\text { Type of intervention } \\
\& \text { theory used }\end{array}$ & Techniques used $\dagger$ & $\begin{array}{l}\text { Behavioural measure \& } \\
\text { type of SSB included }\end{array}$ & Main results on SSB consumption \\
\hline $\begin{array}{l}\text { Bae et al. }(2012)^{(106)} \\
\text { Korea }\end{array}$ & $\begin{array}{l}\text { Evaluate the impact of } \\
\text { governmental nutrition } \\
\text { policies on SSB }\end{array}$ & $\begin{array}{l}\text { Healthy } \\
\text { adolescents }\end{array}$ & $\begin{array}{l}\text { Design: one-group } \\
\text { pre-post } \\
\text { Global rating: moderate }\end{array}$ & $\begin{array}{l}\text { n } 65000 \\
\% \text { o: NR } \\
\text { M age: NR } \\
\text { Middle \& high schools }\end{array}$ & $\begin{array}{l}\text { Educational/behavioural \& } \\
\text { legislative/ } \\
\text { environmental } \\
\text { Theory: N/A }\end{array}$ & $\begin{array}{l}\text { Exp.: } 5,16 \\
\text { Cont.: N/A }\end{array}$ & $\begin{array}{l}\text { Self-administered web-based } \\
\text { survey } \\
\text { SSB: carbonated beverages }\end{array}$ & $\begin{array}{l}\text { Significant reduction in annual } \\
\text { prevalence of SSB consumption } \\
(P<0.05)\end{array}$ \\
\hline $\begin{array}{l}\text { Bauhoff }(2014)^{(73)} \\
\text { USA }\end{array}$ & $\begin{array}{l}\text { Evaluate the impact of a } \\
\text { nutrition policy on SSB }\end{array}$ & $\begin{array}{l}\text { Healthy } \\
\text { adolescents }\end{array}$ & $\begin{array}{l}\text { Design: one-group } \\
\text { pre-post } \\
\text { Global rating: moderate }\end{array}$ & $\begin{array}{l}n 32897 \\
\% \text { o: } 55 \cdot 0 \\
\text { Age (range): } 12-15 \text { years } \\
\text { Grades: } 7 \& 9\end{array}$ & $\begin{array}{l}\text { Legislative/environmental } \\
\text { Theory: N/A }\end{array}$ & $\begin{array}{l}\text { Exp.: } 5 \\
\text { Cont.: N/A }\end{array}$ & $\begin{array}{l}\text { Self-administered } 24 \mathrm{~h} \text { recall } \\
\text { SSB: soda }\end{array}$ & $\begin{array}{l}\text { Only significant reduction in \% of male } \\
\text { adolescents consuming SSB } \\
(P<0.01)\end{array}$ \\
\hline $\begin{array}{l}\text { Blum et al. }(2008)^{(74)} \\
\text { USA }\end{array}$ & $\begin{array}{l}\text { Intervention aimed at } \\
\text { reducing the } \\
\text { availability of SSB in } \\
\text { schools }\end{array}$ & $\begin{array}{l}\text { Healthy } \\
\text { adolescents }\end{array}$ & $\begin{array}{l}\text { Design: quasi- } \\
\quad \text { experimental } \\
\text { Global rating: weak }\end{array}$ & $\begin{array}{l}n 456 \\
\% \text { o: } 40 \cdot 1 \\
\text { M age: } 15 \cdot 8 \text { (sD 0.8) years } \\
\text { Grades: } 9-11\end{array}$ & $\begin{array}{l}\text { Legislative/environmental } \\
\text { Theory: N/A }\end{array}$ & $\begin{array}{l}\text { Exp.: } 5 \\
\text { Cont.: none }\end{array}$ & $\begin{array}{l}\text { Validated self-administered } \\
\text { FFQ } \\
\text { SSB: soda, fruit drinks \& iced } \\
\text { tea }\end{array}$ & $\begin{array}{l}\text { Significant time effect in SSB } \\
\text { consumption for male \& female } \\
\text { adolescents }(P=0.001)\end{array}$ \\
\hline $\begin{array}{l}\text { Bogart et al. }(2001)^{(75)} \\
\text { USA }\end{array}$ & $\begin{array}{l}\text { Healthy eating } \\
\text { intervention }\end{array}$ & $\begin{array}{l}\text { Healthy } \\
\text { adolescents }\end{array}$ & $\begin{array}{l}\text { Design: quasi- } \\
\quad \text { experimental } \\
\text { Global rating: weak }\end{array}$ & $\begin{array}{l}n 425 \\
\% \text { o: } 50.0 \\
\text { M age: } 13.0 \text { (sD } 0.5) \text { years } \\
\text { Grade: } 7\end{array}$ & $\begin{array}{l}\text { Educational/behavioural \& } \\
\text { legislative/ } \\
\text { environmental } \\
\text { Theory: SCT, TPB, EM, DIT }\end{array}$ & $\begin{array}{l}\text { Exp.: } 5,16,54,77,86 \\
\text { Cont.: none }\end{array}$ & $\begin{array}{l}\text { Self-administered survey } \\
\text { SSB: soda, fruit drinks \& sports } \\
\text { drinks }\end{array}$ & $\begin{array}{l}\text { Significant time effect in \% of students } \\
\text { consuming sports/fruit drinks in peer } \\
\text { advocates only }(P<0.05)\end{array}$ \\
\hline $\begin{array}{l}\text { Cassaza }(2006)^{(76)} \\
\text { USA }\end{array}$ & $\begin{array}{l}\text { Healthy eating \& physical } \\
\text { activity intervention }\end{array}$ & $\begin{array}{l}\text { Healthy } \\
\text { adolescents }\end{array}$ & $\begin{array}{l}\text { Design: } \mathrm{RCT} \\
\text { Global rating: weak }\end{array}$ & $\begin{array}{l}n 254 \\
\% \text { d: } 34.2 \\
\text { M age: } 15 \cdot 8 \text { years } \\
\text { Grades: } 9-12\end{array}$ & $\begin{array}{l}\text { Educational/behavioural } \\
\text { Theory: SCT, TPB, TTM, } \\
\text { PM, SM, ET }\end{array}$ & $\begin{array}{l}\text { Exp.: } 16,20,33,46, \\
50,51,59,66,68 \\
80 \\
\text { Cont.: none }\end{array}$ & $\begin{array}{l}\text { Interviewer-administered } 24 \mathrm{~h} \\
\text { recall } \\
\text { SSB: soda }\end{array}$ & $\begin{array}{l}\text { Significant group } \times \text { time effect for soda } \\
\text { consumption }(P<0.01)\end{array}$ \\
\hline $\begin{array}{l}\text { Collins et al. (2014) } \\
\text { Australia }\end{array}$ & $\begin{array}{r}\text { Healthy eating } \\
\text { intervention }\end{array}$ & $\begin{array}{l}\text { Low-SES } \\
\quad \text { adolescent } \\
\text { girls }\end{array}$ & $\begin{array}{l}\text { Design: cluster RCT } \\
\text { Global rating: moderate }\end{array}$ & $\begin{array}{l}n 357 \\
\% \text { o: } 0 \\
\text { M age: } 13.2 \text { years } \\
\text { Secondary schools }\end{array}$ & $\begin{array}{l}\text { Educational/behavioural } \\
\text { Theory: SCT }\end{array}$ & $\begin{array}{l}\text { Exp.: } 16,51,68 \\
\text { Cont.: none }\end{array}$ & $\begin{array}{l}\text { Validated FFQ } \\
\text { SSB: soda, fruit drinks \& cordial } \\
\text { concentrates }\end{array}$ & $\begin{array}{l}\text { Significant time effect for cordial } \\
\text { concentrate \& total SSB consumption } \\
(P<0.05)\end{array}$ \\
\hline $\begin{array}{l}\text { Contento et al. } \\
\quad(2010)^{(77)} \\
\text { USA }\end{array}$ & $\begin{array}{l}\text { Healthy eating \& physical } \\
\text { activity intervention }\end{array}$ & $\begin{array}{l}\text { Low-SES } \\
\text { adolescents }\end{array}$ & $\begin{array}{l}\text { Design: cluster RCT } \\
\text { Global rating: moderate }\end{array}$ & $\begin{array}{l}n 1136 \\
\% \text { d: } 51.0 \\
\text { M age: } 12.0 \text { years } \\
\text { Grade: } 7\end{array}$ & $\begin{array}{l}\text { Educational/behavioural } \\
\text { Theory: SCT, SDT }\end{array}$ & $\begin{array}{l}\text { Exp.: } 2,16,33,46,48, \\
51,59,80 \\
\text { Cont.: none }\end{array}$ & $\begin{array}{l}\text { Validated self-administered } \\
\text { FFQ } \\
\text { SSB: soft drinks, fruit drinks, } \\
\text { sports drinks, iced tea \& } \\
\text { drink mixes }\end{array}$ & $\begin{array}{l}\text { Significant reduction in frequency \& } \\
\quad \text { quantity of } S S B \text { consumption at meals } \\
\& \text { with snacks }(P<0.01)\end{array}$ \\
\hline $\begin{array}{l}\text { Cordeira }(2012)^{(78)} \\
\text { USA }\end{array}$ & $\begin{array}{l}\text { Healthy eating, physical } \\
\text { activity \& tobacco } \\
\text { intervention }\end{array}$ & $\begin{array}{l}\text { Healthy } \\
\text { adolescents }\end{array}$ & $\begin{array}{l}\text { Design: one-group } \\
\text { pre-post } \\
\text { Global rating: weak }\end{array}$ & $\begin{array}{l}n 38 \\
\% \text { o: } 32.0 \\
\text { Age (range): } 13-18 \text { years } \\
\text { Grades: } 9-12\end{array}$ & $\begin{array}{l}\text { Educational/behavioural } \\
\text { Theory: SCT }\end{array}$ & $\begin{array}{l}\text { Exp.: } 2,16,33,46, \\
\quad 68,77 \\
\text { Cont.: N/A }\end{array}$ & $\begin{array}{l}\text { Self-administered survey } \\
\text { SSB: soft drinks \& fruit drinks }\end{array}$ & $\begin{array}{l}\text { No significant reduction in SSB } \\
\text { consumption }\end{array}$ \\
\hline $\begin{array}{l}\text { Cradock et al. } \\
\quad(2011)^{(79)} \\
\text { USA }\end{array}$ & $\begin{array}{l}\text { Evaluate the impact of a } \\
\text { school district policy on } \\
\text { SSB }\end{array}$ & $\begin{array}{l}\text { Healthy } \\
\text { adolescents }\end{array}$ & $\begin{array}{l}\text { Design: quasi- } \\
\quad \text { experimental } \\
\text { Global rating: moderate }\end{array}$ & $\begin{array}{l}n 2091 \\
\% \text { o: } 50 \cdot 7 \\
\text { Age (range): } 15-19 \text { years } \\
\text { Grades: } 9-12\end{array}$ & $\begin{array}{l}\text { Legislative/environmental } \\
\text { Theory: N/A }\end{array}$ & $\begin{array}{l}\text { Exp.: } 5 \\
\text { Cont.: none }\end{array}$ & $\begin{array}{l}\text { Interviewer-administered } 24 \mathrm{~h} \\
\text { recall \& } 7 \mathrm{~d} \text { recall } \\
\text { SSB: soda \& fruit drinks }\end{array}$ & $\begin{array}{l}\text { Significant reduction in total SSB } \\
\text { consumption }(P<0.001)\end{array}$ \\
\hline $\begin{array}{l}\text { Cullen et al. }(2008)^{(80)} \\
\text { USA }\end{array}$ & $\begin{array}{l}\text { Evaluate the impact of a } \\
\text { school nutrition policy } \\
\text { on SBB }\end{array}$ & $\begin{array}{l}\text { Healthy } \\
\text { adolescents }\end{array}$ & $\begin{array}{l}\text { Design: one-group } \\
\text { pre-post } \\
\text { Global rating: strong }\end{array}$ & $\begin{array}{l}n 2671 \\
\% \text { o: NR } \\
\text { Age: NR } \\
\text { Grades: 6-8 }\end{array}$ & $\begin{array}{l}\text { Legislative/environmental } \\
\text { Theory: N/A }\end{array}$ & $\begin{array}{l}\text { Exp.: } 5 \\
\text { Cont.: N/A }\end{array}$ & $\begin{array}{l}\text { Validated food records } \\
\text { SSB: soft drinks \& sweet } \\
\text { beverages }\end{array}$ & $\begin{array}{l}\text { Significant reduction in SSB consumption } \\
\quad(P<0.005)\end{array}$ \\
\hline $\begin{array}{l}\text { da Silva Vargas et al. } \\
(2011)^{(103)} \\
\text { Brazil }\end{array}$ & $\begin{array}{l}\text { Healthy eating \& physical } \\
\text { activity intervention }\end{array}$ & $\begin{array}{l}\text { Normal \& } \\
\quad \text { overweight } \\
\text { adolescents }\end{array}$ & $\begin{array}{l}\text { Design: quasi- } \\
\quad \text { experimental } \\
\text { Global rating: weak }\end{array}$ & $\begin{array}{l}n 331 \\
\% \text { o: NR } \\
\text { M age: } 13 \cdot 1 \text { years } \\
\text { Grades: } 5 \text { \& } 6\end{array}$ & $\begin{array}{l}\text { Educational/behavioural } \\
\text { Theory: none }\end{array}$ & $\begin{array}{l}\text { Exp.: } 16,33,49,57, \\
59,65 \\
\text { Cont.: none }\end{array}$ & $\begin{array}{l}\text { Self-administered questionnaire } \\
\text { SSB: soda }\end{array}$ & $\begin{array}{l}\text { No significant reduction in soda } \\
\text { consumption in both groups }\end{array}$ \\
\hline $\begin{array}{l}\text { Davis et al. (2007) } \\
\text { USA }\end{array}$ & $\begin{array}{r}\text { Healthy eating } \\
\text { intervention }\end{array}$ & $\begin{array}{l}\text { Overweight } \\
\text { Latina } \\
\text { adolescent } \\
\text { girls }\end{array}$ & $\begin{array}{l}\text { Design: } \mathrm{RCT} \\
\text { Global rating: moderate }\end{array}$ & $\begin{array}{l}n 30 \\
\% \text { o: } 0 \\
\text { M age: } 14.7 \text { years } \\
\text { Grade: } N R\end{array}$ & $\begin{array}{l}\text { Educational/behavioural } \\
\text { Theory: none }\end{array}$ & $\begin{array}{l}\text { Exp.: } 2, \mathbf{4 1}, 46, \mathbf{5 1}, \mathbf{5 4}, \\
\mathbf{5 7 ,} 59 \\
\text { Cont.: } 46\end{array}$ & $\begin{array}{l}3 \mathrm{~d} \text { food record \& validated } \\
\text { interviewer-administered } \\
24 \mathrm{~h} \text { recalls } \\
\text { SSB: soda, fruit drinks, sports } \\
\text { drinks, sweetened tea or } \\
\text { coffee }\end{array}$ & $\begin{array}{l}\text { Significant reduction in SSB consumption } \\
\text { in both groups }(P<0.01)\end{array}$ \\
\hline $\begin{array}{l}\text { Dubuy et al. (2014) }{ }^{(101)} \\
\text { Belgium }\end{array}$ & $\begin{array}{l}\text { Healthy eating \& physical } \\
\text { activity intervention }\end{array}$ & $\begin{array}{l}\text { Low-SES } \\
\text { adolescent } \\
\text { boys }\end{array}$ & $\begin{array}{l}\text { Design: quasi- } \\
\text { experimental } \\
\text { Global rating: weak }\end{array}$ & $\begin{array}{l}n 414 \\
\% \text { o: } 100 \\
\text { M age: } 12 \cdot 3 \text { years } \\
\text { Elementary \& secondary } \\
\text { schools }\end{array}$ & $\begin{array}{l}\text { Educational/behavioural } \\
\text { Theory: ELM }\end{array}$ & $\begin{array}{l}\text { Exp.: } 16,43,63,81 \\
\text { Cont:: none }\end{array}$ & $\begin{array}{l}\text { Validated self-administered } \\
\text { FFQ } \\
\text { SSB: soft drinks }\end{array}$ & $\begin{array}{l}\text { No significant reduction in soft drinks } \\
\text { consumption }\end{array}$ \\
\hline
\end{tabular}


Table 1 Continued

\begin{tabular}{|c|c|c|c|c|c|c|c|c|}
\hline $\begin{array}{l}\text { Authors, year, } \\
\text { reference \& country }\end{array}$ & $\begin{array}{l}\text { Objective of the study or } \\
\text { intervention }\end{array}$ & Population & $\begin{array}{l}\text { Study design \& } \\
\text { quality rating* }\end{array}$ & $\begin{array}{l}\text { Baseline characteristics } \\
\text { of sample }\end{array}$ & $\begin{array}{l}\text { Type of intervention } \\
\& \text { theory used }\end{array}$ & Techniques used $\dagger$ & $\begin{array}{l}\text { Behavioural measure \& } \\
\text { type of SSB included }\end{array}$ & Main results on SSB consumption \\
\hline $\begin{array}{l}\text { Greece }(2011)^{(82)} \\
\text { USA }\end{array}$ & $\begin{array}{r}\text { Healthy eating } \\
\text { intervention }\end{array}$ & $\begin{array}{l}\text { Mainly low-SES } \\
\text { adolescents }\end{array}$ & $\begin{array}{l}\text { Design: quasi- } \\
\quad \text { experimental } \\
\text { Global rating: moderate }\end{array}$ & $\begin{array}{l}n 294 \\
\% \text { o: } 45 \cdot 6 \\
\text { M age: } 12.7 \text { (sD } 0.9) \text { years } \\
\text { Grades: } 6-8\end{array}$ & $\begin{array}{l}\text { Legislative/environmental } \\
\text { Theory: N/A }\end{array}$ & $\begin{array}{l}\text { Exp.: } 5,16,23 \\
\text { Cont:: none }\end{array}$ & $\begin{array}{l}\text { Validated self-administered } \\
\text { FFQ } \\
\text { SSB: soft drinks \& fruit drinks }\end{array}$ & $\begin{array}{l}\text { Significant group } \times \text { time effect on SSB } \\
\quad \text { consumption }(P=0.03)\end{array}$ \\
\hline $\begin{array}{l}\text { Haerens et al. } \\
(2006)^{(102)} \\
\text { Belgium }\end{array}$ & $\begin{array}{l}\text { Healthy eating \& physical } \\
\text { activity intervention }\end{array}$ & $\begin{array}{l}\text { Healthy } \\
\text { adolescents }\end{array}$ & $\begin{array}{l}\text { Design: RCT } \\
\text { Global rating: strong }\end{array}$ & $\begin{array}{l}n 2840 \\
\% \text { o: } 63.4 \\
\text { M age: } 13 \cdot 1 \text { (sD } 0.8 \text { ) years } \\
\text { Grades: } 7 \text { \& } 8\end{array}$ & $\begin{array}{l}\text { Educational/behavioural \& } \\
\text { legislative/ } \\
\text { environmental } \\
\text { Theory: TPB, TTM }\end{array}$ & $\begin{array}{l}\text { Exp.: } 5,16,68 \\
\text { Cont.: none }\end{array}$ & $\begin{array}{l}\text { Validated self-administered } \\
\text { FFQ } \\
\text { SSB: soft drinks }\end{array}$ & $\begin{array}{l}\text { No significant reduction in soft drinks } \\
\text { consumption }\end{array}$ \\
\hline $\begin{array}{l}\text { Jones et al. (2014) } \\
\text { USA }\end{array}$ & $\begin{array}{l}\text { Healthy eating \& physical } \\
\text { activity intervention }\end{array}$ & $\begin{array}{l}\text { Normal \& } \\
\quad \text { overweight } \\
\text { adolescents }\end{array}$ & $\begin{array}{l}\text { Design: one-group } \\
\text { pre-post } \\
\text { Global rating: weak }\end{array}$ & $\begin{array}{l}n 336 \\
\% \text { d: } 40.5 \\
\text { M age: } 14.3 \text { years } \\
\text { Grade: } 9\end{array}$ & $\begin{array}{l}\text { Educational/behavioural } \\
\text { Theory: none }\end{array}$ & $\begin{array}{l}\text { Exp.: 2, } 16,33,41,46 \\
49,51,52,54,59, \\
66,68 \\
\text { Cont.: N/A }\end{array}$ & $\begin{array}{l}\text { Self-administered survey } \\
\text { SSB: soda }\end{array}$ & $\begin{array}{l}\text { Significant increase in soda consumption } \\
\text { among adolescents in the healthy } \\
\text { habits track }(P=0.001) \\
\text { Significant decrease in soda } \\
\text { consumption among adolescents in } \\
\text { the weight management track } \\
(P=0.002)\end{array}$ \\
\hline $\begin{array}{l}\text { Lao }(2011)^{(83)} \\
\text { USA }\end{array}$ & $\begin{array}{l}\text { Healthy eating \& physical } \\
\text { activity intervention }\end{array}$ & $\begin{array}{l}\text { Low-SES } \\
\quad \text { adolescents }\end{array}$ & $\begin{array}{l}\text { Design: } \mathrm{RCT} \\
\text { Global rating: weak }\end{array}$ & $\begin{array}{l}n 192 \\
\% \text { \%: } 55 \cdot 7 \\
\text { M age: } 14.9 \text { years } \\
\text { Grades: } 9 \& 10\end{array}$ & $\begin{array}{l}\text { Educational/behavioural } \\
\text { Theory: TTM }\end{array}$ & $\begin{array}{l}\text { Exp.: } 16,33,46,54, \\
59,65,66,68,72 \\
\text { Cont.: none }\end{array}$ & $\begin{array}{l}\text { Self-administered survey } \\
\text { SSB: soda, fruit, sports \& } \\
\text { energy drinks }\end{array}$ & $\begin{array}{l}\text { No significant reduction in SSB } \\
\text { consumption }\end{array}$ \\
\hline $\begin{array}{l}\text { Lo et al. (2008) } \\
\text { Canada }\end{array}$ & $\begin{array}{l}\text { Intervention aimed at } \\
\text { reducing SSB } \\
\text { consumption }\end{array}$ & $\begin{array}{l}\text { Healthy } \\
\text { adolescents }\end{array}$ & $\begin{array}{l}\text { Design: quasi- } \\
\quad \text { experimental } \\
\text { Global rating: weak }\end{array}$ & $\begin{array}{l}n 101 \\
\% \text { o: } 61.4 \\
\text { Mage: } 14 \text { years } \\
\text { Grade: } 9\end{array}$ & $\begin{array}{l}\text { Educational/behavioural } \\
\text { Theory: CTL }\end{array}$ & $\begin{array}{l}\text { Exp.: } 15,16,17,59,63 \\
\text { Cont.: } 15,16,17,59\end{array}$ & $\begin{array}{l}\text { Self-administered questionnaire } \\
\text { SSB: soft drinks, fruit drinks, } \\
\text { sports drinks, iced tea, } \\
\text { sweetened tea \& coffee }\end{array}$ & $\begin{array}{l}\text { Significant reduction in SSB consumption } \\
\text { at the 3-month follow-up in the } \\
\text { experimental group }(P<0.02)\end{array}$ \\
\hline $\begin{array}{l}\text { Malbon }(2012)^{(97)} \\
\text { Canada }\end{array}$ & $\begin{array}{l}\text { Healthy eating \& physical } \\
\text { activity intervention }\end{array}$ & $\begin{array}{l}\text { Healthy } \\
\text { adolescents }\end{array}$ & $\begin{array}{l}\text { Design: one-group } \\
\text { pre-post } \\
\text { Global rating: weak }\end{array}$ & $\begin{array}{l}n 44 \\
\% \text { o: } 48.0 \\
\text { Age: NR } \\
\text { Grade: } 10\end{array}$ & $\begin{array}{l}\text { Educational/behavioural } \\
\text { Theory: SDT }\end{array}$ & $\begin{array}{l}\text { Exp.: } 16,21,46,51, \\
54,66,73,80 \\
\text { Cont.: N/A }\end{array}$ & $\begin{array}{l}\text { Self-administered questionnaire } \\
\text { SSB: soft drinks, fruit drinks, } \\
\text { energy drinks, iced tea \& } \\
\text { slush }\end{array}$ & $\begin{array}{l}\text { No significant reduction in SSB } \\
\text { consumption }\end{array}$ \\
\hline $\begin{array}{l}\text { McGoldrick (2006) } \\
\text { Canada }\end{array}$ & $\begin{array}{l}\text { Evaluate the impact of } \\
\text { governmental nutrition } \\
\text { policies on SSB }\end{array}$ & $\begin{array}{l}\text { Healthy } \\
\text { adolescents }\end{array}$ & $\begin{array}{l}\text { Design: one-group } \\
\text { pre-post } \\
\text { Global rating: moderate }\end{array}$ & $\begin{array}{l}n 703 \\
\% \text { o: } 43 \cdot 5 \\
\text { Age (range): } 10-14 \text { years } \\
\text { Grades: } 6-8\end{array}$ & $\begin{array}{l}\text { Legislative/environmental } \\
\text { Theory: N/A }\end{array}$ & $\begin{array}{l}\text { Exp.: } 5 \\
\text { Cont.: N/A }\end{array}$ & $\begin{array}{l}\text { Validated web } 24 \mathrm{~h} \text { recall \& self- } \\
\text { administered FFQ } \\
\text { SSB: soft drinks, fruit drinks, } \\
\text { sports drinks \& iced tea }\end{array}$ & $\begin{array}{l}\text { Significant increase in volume of SSB } \\
\text { consumed, significant reduction in } \\
\text { frequency of cola consumption \& } \\
\text { significant increase in \% of } \\
\text { adolescents who consume SSB } \\
(P<0.001)\end{array}$ \\
\hline $\begin{array}{l}\text { Nanney et al. } \\
(2014)^{(84)} \\
\text { USA }\end{array}$ & $\begin{array}{l}\text { Evaluate the impact of } \\
\text { school policies on SSB }\end{array}$ & $\begin{array}{l}\text { Healthy } \\
\text { adolescents }\end{array}$ & $\begin{array}{l}\text { Design: one-group } \\
\text { pre-post } \\
\text { Global rating: moderate }\end{array}$ & $\begin{array}{l}n 18881 \\
\% \text { o: NR } \\
\text { Age: NR } \\
\text { Grades: } 9 \text { \& } 12\end{array}$ & $\begin{array}{l}\text { Legislative/environmental } \\
\text { Theory: N/A }\end{array}$ & $\begin{array}{l}\text { Exp.: } 5 \\
\text { Cont.: N/A }\end{array}$ & $\begin{array}{l}\text { Self-administered questionnaire } \\
\text { SSB: soda \& sports drinks }\end{array}$ & $\begin{array}{l}\text { Significant reduction in SSB consumption } \\
\qquad(P=0.04)\end{array}$ \\
\hline $\begin{array}{l}\text { Nanney et al. } \\
(2016)^{(95)} \\
\text { USA }\end{array}$ & $\begin{array}{l}\text { Evaluate the impact of } \\
\text { school policies on SSB }\end{array}$ & $\begin{array}{l}\text { Healthy } \\
\text { adolescents }\end{array}$ & $\begin{array}{l}\text { Design: one-group } \\
\text { pre-post } \\
\text { Global rating: moderate }\end{array}$ & $\begin{array}{l}n 7237 \\
\% \text { o: NR } \\
\text { Age: NR } \\
\text { Grade: } 9\end{array}$ & $\begin{array}{l}\text { Legislative/environmental } \\
\text { Theory: N/A }\end{array}$ & $\begin{array}{l}\text { Exp.: } 5 \\
\text { Cont.: N/A }\end{array}$ & $\begin{array}{l}\text { Self-administered questionnaire } \\
\text { SSB: soda \& sports drinks }\end{array}$ & $\begin{array}{l}\text { Significant reduction in soda } \\
\text { consumption }(P<0.05)\end{array}$ \\
\hline $\begin{array}{l}\text { Neumark-Sztainer } \\
\text { et al. }(2010)^{(85)} \\
\text { USA }\end{array}$ & $\begin{array}{l}\text { Healthy eating \& physical } \\
\text { activity intervention }\end{array}$ & $\begin{array}{l}\text { Low-SES normal } \\
\& \text { overweight/ } \\
\text { obese } \\
\text { adolescent } \\
\text { girls }\end{array}$ & $\begin{array}{l}\text { Design: } \mathrm{RCT} \\
\text { Global rating: weak }\end{array}$ & $\begin{array}{l}n 356 \\
\% \text { o: } 0 \\
\text { M age: } 15.8 \text { years } \\
\text { High schools }\end{array}$ & $\begin{array}{l}\text { Educational/behavioural } \\
\text { Theory: SCT, TTM }\end{array}$ & $\begin{array}{l}\text { Exp.: 2, } 16,41,46,57, \\
59,68, \\
\text { Cont.: none }\end{array}$ & $\begin{array}{l}\text { Interviewer-administered } 24 \mathrm{~h} \\
\text { recall } \\
\text { SSB: soda, fruit drinks, sports } \\
\text { drinks, sweetened tea \& } \\
\text { sweetened coffee }\end{array}$ & $\begin{array}{l}\text { No significant reduction in SSB } \\
\text { consumption at follow-up }\end{array}$ \\
\hline $\begin{array}{l}\text { Pbert et al. }(2013)^{(87)} \\
\text { USA }\end{array}$ & $\begin{array}{l}\text { Healthy eating \& physical } \\
\text { activity intervention }\end{array}$ & $\begin{array}{l}\text { Overweight \& } \\
\text { obese } \\
\text { adolescents }\end{array}$ & $\begin{array}{l}\text { Design: cluster RCT } \\
\text { Global rating: moderate }\end{array}$ & $\begin{array}{l}n 82 \\
\% \text { o: } 30.5 \\
\text { M age: } 15 \cdot 8 \text { years } \\
\text { Grades: } 9-11\end{array}$ & $\begin{array}{l}\text { Educational/behavioural } \\
\text { Theory: SCT }\end{array}$ & $\begin{array}{l}\text { Exp.: 2, 16, 46, 47, 51, } \\
\quad 66,72 \\
\text { Cont.: } 16\end{array}$ & $\begin{array}{l}\text { Validated telephone- } \\
\text { administered } 24 \mathrm{~h} \text { recall } \\
\text { SSB: soda \& sugary drinks }\end{array}$ & $\begin{array}{l}\text { No significant reduction in SSB } \\
\text { consumption }\end{array}$ \\
\hline
\end{tabular}


Table 1 Continued

\begin{tabular}{|c|c|c|c|c|c|c|c|c|}
\hline $\begin{array}{l}\text { Authors, year, } \\
\text { reference \& country }\end{array}$ & $\begin{array}{l}\text { Objective of the study or } \\
\text { intervention }\end{array}$ & Population & $\begin{array}{l}\text { Study design \& } \\
\text { quality rating }\end{array}$ & $\begin{array}{l}\text { Baseline characteristics } \\
\text { of sample }\end{array}$ & $\begin{array}{l}\text { Type of intervention } \\
\& \text { theory used }\end{array}$ & Techniques used $\dagger$ & $\begin{array}{l}\text { Behavioural measure \& } \\
\text { type of SSB included }\end{array}$ & Main results on SSB consumption \\
\hline $\begin{array}{l}\text { Singhal et al. } \\
\quad(2010)^{(105)} \\
\text { India }\end{array}$ & $\begin{array}{l}\text { Healthy eating \& physical } \\
\text { activity intervention }\end{array}$ & $\begin{array}{l}\text { Healthy } \\
\text { adolescents }\end{array}$ & $\begin{array}{l}\text { Design: } \mathrm{RCT} \\
\text { Global rating: moderate }\end{array}$ & $\begin{array}{l}n 209 \\
\% \text { d: } 59.8 \\
\text { M age: } 16.0 \text { years } \\
\text { Grade: } 11\end{array}$ & $\begin{array}{l}\text { Educational/behavioural } \\
\text { Theory: none }\end{array}$ & $\begin{array}{l}\text { Exp.: } 2,5,16,33,48, \\
\quad 59 \\
\text { Cont.: none }\end{array}$ & $\begin{array}{l}\text { Self-administered questionnaire } \\
\text { SSB: soft drinks }\end{array}$ & $\begin{array}{l}\text { Significant reduction in \% of adolescents } \\
\text { consuming soft drinks at least } 3 \text { times/ } \\
\text { week in the experimental group } \\
(P=0.001)\end{array}$ \\
\hline $\begin{array}{l}\text { Smith et al. (2014) } \\
\text { Australia }\end{array}$ & $\begin{array}{l}\text { Healthy eating \& physical } \\
\text { activity intervention }\end{array}$ & $\begin{array}{l}\text { Low-SES } \\
\text { adolescent } \\
\text { boys at risk } \\
\text { for obesity }\end{array}$ & $\begin{array}{l}\text { Design: cluster } \mathrm{RCT} \\
\text { Global rating: weak }\end{array}$ & $\begin{array}{l}n 361 \\
\% \delta \text { o: } 100 \\
\text { M age: } 12.7 \text { (sD } 0.5) \text { years } \\
\text { Secondary schools }\end{array}$ & $\begin{array}{l}\text { Educational/behavioural } \\
\text { Theory: SCT, SDT }\end{array}$ & $\begin{array}{l}\text { Exp.: } 2,16,33,46,51, \\
59,63,66,68,72, \\
\quad 77 \\
\text { Cont.: none }\end{array}$ & $\begin{array}{l}\text { Self-administered questionnaire } \\
\text { SSB: NR }\end{array}$ & $\begin{array}{l}\text { Significant group } \times \text { time effect on SSB } \\
\text { consumption }(P=0.01)\end{array}$ \\
\hline $\begin{array}{l}\text { Smith \& Holloman } \\
(2014)^{(88)} \\
\text { USA }\end{array}$ & $\begin{array}{l}\text { Intervention aimed at } \\
\quad \text { reducing SSB } \\
\text { consumption }\end{array}$ & $\begin{array}{l}\text { Mainly low-SES } \\
\text { adolescents }\end{array}$ & $\begin{array}{l}\text { Design: one-group } \\
\text { pre-post } \\
\text { Global rating: weak }\end{array}$ & $\begin{array}{l}n 186 \\
\% \text { o: } 39 \cdot 2 \\
\text { M age: } 15 \cdot 9 \text { (sD } 1 \cdot 8) \text { years } \\
\text { Grades: } 9-12\end{array}$ & $\begin{array}{l}\text { Educational/behavioural } \\
\text { Theory: none }\end{array}$ & $\begin{array}{l}\text { Exp.: } 16,33,65 \\
\text { Cont.: N/A }\end{array}$ & $\begin{array}{l}\text { Self-administered questionnaire } \\
\text { \& food record } \\
\text { SSB: soft drinks, fruit drinks, } \\
\text { sports drinks, energy drinks, } \\
\text { sweetened tea \& coffee }\end{array}$ & $\begin{array}{l}\text { Significant reduction in frequency \& } \\
\text { quantity of SSB consumed at } 30 \mathrm{~d} \\
\text { follow-up }(P<0.05)\end{array}$ \\
\hline $\begin{array}{l}\text { Teufel \& Ritenbaugh } \\
\quad(1998)^{(89)} \\
\text { USA }\end{array}$ & $\begin{array}{l}\text { Healthy eating \& physical } \\
\text { activity intervention }\end{array}$ & $\begin{array}{l}\text { Native American } \\
\text { adolescents }\end{array}$ & $\begin{array}{l}\text { Design: one-group } \\
\text { pre--post } \\
\text { Global rating: weak }\end{array}$ & $\begin{array}{l}n 119 \\
\% \text { o: } 44.5 \\
\text { M age: } 17 \cdot 2 \text { (sD } 4.0) \text { years } \\
\text { Grades: } 9-12\end{array}$ & $\begin{array}{l}\text { Educational/behavioural \& } \\
\text { legislative/ } \\
\text { environmental } \\
\text { Theory: none }\end{array}$ & $\begin{array}{l}\text { Exp.: } 5,16,33,68 \\
\text { Cont.: N/A }\end{array}$ & $\begin{array}{l}24 \mathrm{~h} \text { recall } \\
\text { SSB: soft drinks \& fruit drinks }\end{array}$ & $\begin{array}{l}\text { Significant reduction in \% of SSB } \\
\text { consumed }(P<0.05)\end{array}$ \\
\hline $\begin{array}{l}\text { Thiele \& Boushey } \\
\qquad(1989)^{(90)} \\
\text { USA }\end{array}$ & $\begin{array}{l}\text { Intervention aimed at } \\
\text { reducing SSB } \\
\text { consumption }\end{array}$ & $\begin{array}{l}\text { Eskimo } \\
\quad \text { adolescents }\end{array}$ & $\begin{array}{l}\text { Design: quasi- } \\
\quad \text { experimental } \\
\text { Global rating: weak }\end{array}$ & $\begin{array}{l}n 374 \\
\% \text { o: NR } \\
\text { Age: NR } \\
\text { Grades: } 7-12\end{array}$ & $\begin{array}{l}\text { Educational/behavioural } \\
\text { Theory: none }\end{array}$ & $\begin{array}{l}\text { Exp.: } 16 \\
\text { Cont.: none }\end{array}$ & $\begin{array}{l}\text { Interviewer-administered } 24 \mathrm{~h} \\
\text { recall } \\
\text { SSB: soft drinks \& fruit drinks }\end{array}$ & $\begin{array}{l}\text { Significant reduction in SSB consumption } \\
\text { in one of the two experimental groups } \\
(P=0.001)\end{array}$ \\
\hline $\begin{array}{l}\text { Whittemore et al. } \\
(2013)^{\left.()_{11}\right)} \\
\text { USA }\end{array}$ & $\begin{array}{l}\text { Healthy eating \& physical } \\
\text { activity intervention }\end{array}$ & $\begin{array}{l}\text { Healthy } \\
\text { adolescents }\end{array}$ & $\begin{array}{l}\text { Design: } \mathrm{RCT} \\
\text { Global rating: weak }\end{array}$ & $\begin{array}{l}n 384 \\
\% \text { o: } 38.0 \\
\text { M age: } 15.3 \text { (sD } 0.7) \text { years } \\
\text { High schools }\end{array}$ & $\begin{array}{l}\text { Educational/behavioural } \\
\text { Theory: SLT, TIT }\end{array}$ & $\begin{array}{l}\text { Exp.: } 2,16,41,46,51, \\
54,57,63,66,68, \\
72,82 \\
\text { Cont.: } 16,46,51,54, \\
57,63,66,68 \\
72,82\end{array}$ & $\begin{array}{l}\text { Self-administered questionnaire } \\
\text { SSB: soda \& fruit drinks }\end{array}$ & $\begin{array}{l}\text { Significant reduction in SSB consumption } \\
\text { in both groups }(P<0.01)\end{array}$ \\
\hline $\begin{array}{l}\text { Winett et al. }(1999)^{(92)} \\
\text { USA }\end{array}$ & $\begin{array}{l}\text { Healthy eating \& physical } \\
\text { activity intervention }\end{array}$ & $\begin{array}{l}\text { Adolescent girls } \\
\text { from } \\
\text { medically } \\
\text { underserved } \\
\text { areas }\end{array}$ & $\begin{array}{l}\text { Design: quasi- } \\
\quad \text { experimental } \\
\text { Global rating: weak }\end{array}$ & $\begin{array}{l}n 180 \\
\% \text { o: } 0 \\
\text { M age: } 15.4 \text { years } \\
\text { Grades: } 9 \& 10\end{array}$ & $\begin{array}{l}\text { Educational/behavioural } \\
\text { Theory: SCT }\end{array}$ & $\begin{array}{l}\text { Exp.: } 16,46,47,51 \text {, } \\
54,66,71 \\
\text { Cont.: none }\end{array}$ & $\begin{array}{l}\text { Self-administered } 24 \mathrm{~h} \text { recall \& } \\
\text { FFQ } \\
\text { SSB: soda }\end{array}$ & $\begin{array}{l}\text { Significant group } \times \text { time effect on soda } \\
\text { consumption }(P<0.05)\end{array}$ \\
\hline $\begin{array}{l}\text { Wing et al. (2015) } \\
\text { China }\end{array}$ & $\begin{array}{l}\text { Intervention aimed at } \\
\text { promoting sleep }\end{array}$ & $\begin{array}{l}\text { Healthy } \\
\text { adolescents }\end{array}$ & $\begin{array}{l}\text { Design: cluster RCT } \\
\text { Global rating: weak }\end{array}$ & $\begin{array}{l}\text { n } 5219 \\
\% \text { o: } 39.0 \\
\text { M age: } 14.7 \text { years } \\
\text { Grades: } 7-11\end{array}$ & $\begin{array}{l}\text { Educational/behavioural } \\
\text { Theory: none }\end{array}$ & $\begin{array}{l}\text { Exp.: } 16,33,41,50,51 \\
\text { Cont.: none }\end{array}$ & $\begin{array}{l}\text { Self-administered questionnaire } \\
\text { SSB: energy drinks }\end{array}$ & $\begin{array}{l}\text { Significant difference in incidence of } \\
\text { energy drinks consumption in } \\
\text { experimental } v \text {. control group } \\
(P<0.05)\end{array}$ \\
\hline $\begin{array}{l}\text { Woodward-Lopez } \\
\text { et al. }(2010)^{(93)} \\
\text { USA }\end{array}$ & $\begin{array}{l}\text { Evaluate the impact of } \\
\text { school policies on SSB }\end{array}$ & $\begin{array}{l}\text { Low-SES } \\
\quad \text { adolescents }\end{array}$ & $\begin{array}{l}\text { Design: one-group } \\
\text { pre-post } \\
\text { Global rating: weak }\end{array}$ & $\begin{array}{l}n 3527 \\
\% \text { o: NR } \\
\text { Age: NR } \\
\text { Grades: } 7 \& 9\end{array}$ & $\begin{array}{l}\text { Legislative/environmental } \\
\text { Theory: N/A }\end{array}$ & $\begin{array}{l}\text { Exp.: } 5 \\
\text { Cont.: N/A }\end{array}$ & $\begin{array}{l}\text { Self-administered questionnaire } \\
\text { SSB: soda \& sports drinks }\end{array}$ & $\begin{array}{l}\text { Significant reduction in \% of adolescents } \\
\text { consuming sodas at school }(P<0.01)\end{array}$ \\
\hline $\begin{array}{l}\text { Wordell et al. } \\
(2012)^{(94)} \\
\text { USA }\end{array}$ & $\begin{array}{l}\text { Evaluate the impact of } \\
\text { changes in the school } \\
\text { food environment }\end{array}$ & $\begin{array}{l}\text { Low-SES } \\
\quad \text { adolescents }\end{array}$ & $\begin{array}{l}\text { Design: quasi- } \\
\text { experimental } \\
\text { Global rating: weak }\end{array}$ & $\begin{array}{l}n 2292 \\
\% \text { o: } 51.0 \\
\text { Age: NR } \\
\text { Grades: } 7 \text { \& } 8\end{array}$ & $\begin{array}{l}\text { Legislative/environmental } \\
\text { Theory: N/A }\end{array}$ & $\begin{array}{l}\text { Exp.: } 5 \\
\text { Cont.: none }\end{array}$ & $\begin{array}{l}\text { Self-administered FFQ } \\
\text { SSB: energy drinks \& sweet } \\
\text { drinks }\end{array}$ & $\begin{array}{l}\text { No significant reduction in SSB } \\
\text { consumption }\end{array}$ \\
\hline $\begin{array}{l}\text { Yildirim et al. } \\
\quad(2013)^{(107)} \\
\text { The Netherlands }\end{array}$ & $\begin{array}{l}\text { Healthy eating \& physical } \\
\text { activity intervention }\end{array}$ & $\begin{array}{l}\text { Low- } \\
\text { educational- } \\
\text { level } \\
\text { adolescents }\end{array}$ & $\begin{array}{l}\text { Design: } \mathrm{RCT} \\
\text { Global rating: weak }\end{array}$ & $\begin{array}{l}n 1108 \\
\% \text { o d: } 46 \cdot 7 \\
\text { M age: } 12.8 \text { years } \\
\text { Grade: } \mathrm{NR}\end{array}$ & $\begin{array}{l}\text { Educational/behavioural \& } \\
\text { legislative/ } \\
\text { environmental } \\
\text { Theory: SRT, EnRG } \\
\text { framework (DPT, } \\
\text { ANGELO model, TPB, } \\
\text { Habit theory) }\end{array}$ & $\begin{array}{l}\text { Exp.: } 2,5,16,46,48, \\
51,54,57,63,66, \\
68,73 \\
\text { Cont.: none }\end{array}$ & $\begin{array}{l}\text { Self-administered questionnaire } \\
\text { SSB: soft drinks, lemonade, } \\
\text { energy drinks \& iced tea }\end{array}$ & $\begin{array}{l}\text { Significant reduction in SSB consumption } \\
(P<0.001)\end{array}$ \\
\hline
\end{tabular}

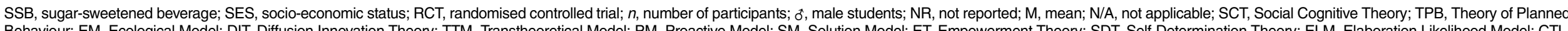

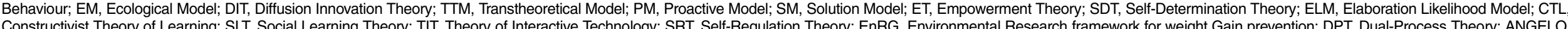

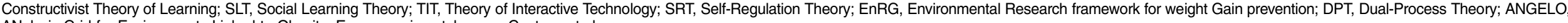
ANalysis Grid for Environments Linked to Obesity; Exp., experimental group; Cont., control group.

${ }^{*}$ Global rating of the quality of studies was performed using the Effective Public Health Practice Project (EPHPP) Quality Assessment Tool for Quantitative Studies ${ }^{(71)}$.

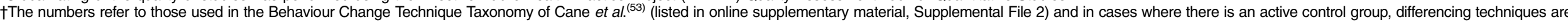
presented in bold font. 
or healthy eating alone $\left(\begin{array}{ll}k & 4\end{array}\right)^{(75,81,82,99)}$. One study also included tobacco prevention ${ }^{(78)}$. Ten interventions (27.8\%) had the objective of evaluating the impact of nutrition policies in schools or changes in the school environment, such as reduced availability of $\operatorname{SSB}^{(73,74,79,80,84,93-95,98,106)}$. Only four interventions (11.1\%) were specifically aimed at reducing SSB consumption and increasing water consumption $^{(86,88,90,96)}$. Finally, one intervention was aimed at promoting sleep and included reducing energy drinks consumption as a means of achieving this goal $^{(104)}$.

Twenty interventions $(55 \cdot 6 \%)$ were classified as educational/behavioural $^{(38,76-78,81,83,85,87,88,90-92,96,97,99-101,103-105)}$, while all the studies on school policies and environmental changes ( $k$ 10) were classified as legislative/environmental interventions $^{(38,73,74,79,80,84,93-95,98)}$ (see Table 2). Only six interventions (16.7\%) included both an educational/behavioural component and a legislative/environmental component ${ }^{(75,86,89,102,106,107)}$.

\section{Characteristics of participants}

About half of the interventions $(47 \cdot 2 \%, k 17)$ were conducted among healthy adolescents ${ }^{(73-76,78-80,84,91,95-98,102,104-106)}$. Thirteen studies (36.1\%) targeted adolescents whose parents had a low $\operatorname{SES}^{(38,77,82,85,86,88,93,94,99-101)}$, who had a low educational level ${ }^{(107)}$ or who were living in medically underserved areas ${ }^{(92)}$. Four studies targeted adolescent girls only ${ }^{(81,85,92,99)}$ and two adolescent boys only ${ }^{(100,101)}$. Three studies targeted a mix of normal-weight and overweight adolescents ${ }^{(38,85,103)}$, two studies targeted overweight/obese adolescents ${ }^{(81,87)}$ and another targeted adolescents at risk for obesity ${ }^{(100)}$. Finally, three studies targeted specific ethnic minorities in the USA, such as Latina girls ${ }^{(81)}$, Native Americans $^{(89)}$ and Inuits ${ }^{(90)}$. It is worth noting that some studies targeted adolescents with multiple sociodemographic characteristics, such as overweight adolescents whose parents had a low SES, which explains why the number of studies in this section exceeds the number of studies included in the review.

Among the twenty-nine studies reporting information on the sex of participants ${ }^{(38,73-79,81-83,85-89,91,92,94,96-102,104,105,107)}$, $43.6 \%$ of samples were comprised of adolescent boys. The pooled mean age of the twenty-four studies reporting age was $14 \cdot 3$ years $38,74-77,81-83,85-89,91,92,96,99-105,107)$. Finally, twenty-eight studies reported information on the level of education of their participants ${ }^{(38,73-80,82-84,86-90,92-98,102-105)}$. The range of education was from grades 6 to 12. Depending on the country where the study was conducted, this referred to either elementary, middle or high/secondary schools or a mix of these schools.

\section{Behavioural measures of sugar-sweetened beverage consumption}

Less than a third (27.8\%) of studies ( $k$ 10) used a validated tool to measure SSB consumption $(74,77,80-82,87,98,99,101,102)$ and three out of four of the instruments $(75 \cdot 0 \%, k 27)$ were
Table 2 Efficacy of interventions included in the present systematic review according to their type

\begin{tabular}{|c|c|c|c|}
\hline \multirow[b]{2}{*}{ Reference } & \multicolumn{3}{|c|}{ SSB consumption } \\
\hline & $\begin{array}{l}\text { Significant } \\
\text { reduction }\end{array}$ & $\begin{array}{l}\text { No significant } \\
\text { reduction }\end{array}$ & $\begin{array}{l}\text { Significant } \\
\text { increase }\end{array}$ \\
\hline \multicolumn{4}{|c|}{ Educational/behavioural studies $(k 20)$} \\
\hline Cassaza $^{(76)}$ & $\checkmark$ & & \\
\hline Collins et al. ${ }^{(99)}$ & $\checkmark$ & & \\
\hline $\begin{array}{l}\text { Contento } \\
\text { et al. }\end{array}$ & $\checkmark$ & & \\
\hline Cordeira $^{(78)}$ & & $\checkmark$ & \\
\hline $\begin{array}{l}\text { da Silva Vargas } \\
\text { et al. }{ }^{(103)}\end{array}$ & & $\checkmark$ & \\
\hline Davis et al. ${ }^{(81) *}$ & $\checkmark$ & & \\
\hline Dubuy et al. ${ }^{(101)}$ & & $\checkmark$ & \\
\hline Jones et al. ${ }^{(38)} \dagger$ & $\checkmark$ & & $\checkmark$ \\
\hline $\mathrm{Lao}^{(83)}$ & & $\checkmark$ & \\
\hline Malbon ${ }^{(97)}$ & $\checkmark$ & $\checkmark$ & \\
\hline $\begin{array}{l}\text { Neumark- } \\
\text { Sztainer et al. }{ }^{(85)}\end{array}$ & & $\checkmark$ & \\
\hline Pbert et al. ${ }^{(87)}$ & & $\checkmark$ & \\
\hline Singhal et al. ${ }^{(105)}$ & $\checkmark$ & & \\
\hline Smith et al. ${ }^{(100)}$ & $\checkmark$ & & \\
\hline $\begin{array}{l}\text { Smith \& } \\
\text { Holloman }\end{array}$ & $\checkmark$ & & \\
\hline $\begin{array}{l}\text { Thiele \& } \\
\text { Boushey } \\
{ }^{(90)} \ddagger\end{array}$ & $\checkmark$ & & \\
\hline 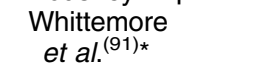 & $\checkmark$ & & \\
\hline Winnet et al. ${ }^{(92)}$ & $\checkmark$ & & \\
\hline Wing et al. ${ }^{(104)}$ & $\checkmark$ & & \\
\hline Total & 13 & 7 & 1 \\
\hline \multicolumn{4}{|c|}{ Legislative/environmental studies $(k 10)$} \\
\hline Bauhoff $^{(73)} \neq$ & $\lambda$ & & \\
\hline Blum et al. ${ }^{(74) \star}$ & $\checkmark$ & & \\
\hline Cradock et al. ${ }^{(79)}$ & $\checkmark$ & & \\
\hline Cullen et al. ${ }^{(80)}$ & $\checkmark$ & & \\
\hline Greece $^{(82)}$ & $\checkmark$ & & \\
\hline McGoldrick ${ }^{(98)} \S$ & $\checkmark$ & & $\checkmark$ \\
\hline Nanney et al. ${ }^{(84)}$ & $\checkmark$ & & \\
\hline Nanney et al. & $\checkmark$ & & \\
\hline $\begin{array}{l}\text { Woodward-Lopez } \\
\text { et al. }\end{array}$ & $\checkmark$ & & \\
\hline Wordell et al. ${ }^{(94)}$ & & $\checkmark$ & \\
\hline Total & 9 & 1 & 1 \\
\hline \multicolumn{4}{|c|}{ Educational/behavioural \& legislative/environmental studies $(k 6)$} \\
\hline $\begin{array}{l}\text { Bae } \\
\text { et al. }{ }^{(106)}\end{array}$ & 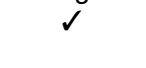 & & \\
\hline $\begin{array}{l}\text { Bogart } \\
\text { et al. } .^{(75) \star}, \neq\end{array}$ & $\checkmark$ & & \\
\hline $\begin{array}{l}\text { Haerens } \\
\text { et }^{\text {el. }}{ }^{(102)}\end{array}$ & & $\checkmark$ & \\
\hline Patel et al. ${ }^{(86)}$ & & $\checkmark$ & \\
\hline $\begin{array}{l}\text { Teufel \& } \\
\text { Ritenbaugh }\end{array}$ & $\checkmark$ & & \\
\hline Yildirim et al. ${ }^{(107)}$ & $\checkmark$ & & \\
\hline Total & 4 & 2 & 0 \\
\hline
\end{tabular}

SSB, sugar-sweetened beverage; $k$, number of studies.

* Significant time effect only or significant reduction of SSB consumption in both the experimental and the control group.

†Significant reduction of SSB consumption in half of the experimental group (peer advocates) and significant increase of SSB consumption in the other half of the experimental group (non-peer advocates).

$\ddagger$ Significant reduction of SSB consumption in half of the experimental group and no significant reduction of SSB consumption in the other half of the experimental group.

§Significant reduction in frequency of cola consumption, but significant increases in volume of SBB consumed and in percentage of adolescents who consume SSB. 
self-administered ${ }^{(38,73-75,77,78,82-84,86,88,91-98,100-107)}$. Among them, two studies used web-based questionnaires, such as a web-based survey ${ }^{(106)}$ and a web 24 h recall $^{(98)}$. Three studies did not specify the mode of delivery of their behavioural measure ${ }^{(80,89,99)}$. The most common method for measuring SSB consumption was a survey or questionnaire $(50 \cdot 0 \%, k 18)^{(38,75,78,83,84,86,88,91,93,95-97,100,103-107)}$, followed by $24 \mathrm{~h}$ recalls $(27 \cdot 8 \%, k 10)^{(73,76,79,81,84,86,88,89,91,97)}$, an FFQ $(25 \cdot 0 \%, k 9)^{(74,77,82,91,93,97,98,100,101)}$ and food records $(8.3 \%, k 3)^{(80,81,88)}$. Among the studies that used $24 \mathrm{~h}$ recalls, $60 \cdot 0 \%(k, 6)$ chose interviewer-administered ${ }^{(76,79,81,85,90)}$ or telephone-administered $^{(87)} 24 \mathrm{~h}$ recalls for assessing SSB consumption. Four studies (11.1\%) used multiple selfreported tools, such as a $3 \mathrm{~d}$ food record and $24 \mathrm{~h}$ recalls ${ }^{(81)}$, a $24 \mathrm{~h}$ recall and an $\mathrm{FFQ}^{(92,98)}$, and a questionnaire and a food record ${ }^{(88)}$.

More than $90 \%(91.7 \%)$ of studies ( $k$ 33) included soft drinks such as soda in their definition of $\mathrm{SBB}^{(38,73-93,95-99,101-103,105-107)}$. Among the studies that did not measure soft drinks, one did not specify the type of beverages included in its definition of $\mathrm{SSB}^{(100)}$, another only considered energy drinks ${ }^{(104)}$ and one included energy drinks and sweet drinks without specifying if soft drinks were included in the latter category of beverages ${ }^{(96)}$. Seventeen studies $(47 \cdot 2 \%)$ included fruit drinks in their SSB definition ${ }^{(74,75,77-79,81-83,85,88-91,96-99)}$ and twelve studies $(33 \cdot 3 \%)$ sports drinks ${ }^{(75,77,81,83-86,88,93,95,97,98)}$. Nine studies (25.0\%) included either iced tea or sweetened tea and coffee $^{(74,77,81,85,88,96-98,107)}$. Six studies $(16 \cdot 7 \%)$ included energy drinks ${ }^{(83,88,94,97,104,107)}$ and four studies (11.1\%) included other types of SSB such as cordial concentrates ${ }^{(99)}$, drink mixes ${ }^{(77)}$, slush ${ }^{(97)}$ and lemonade ${ }^{(107)}$. Finally, three studies (8.3\%) had a general 'sweet drinks' category $(80,87,94)$.

Nine studies $(25 \cdot 0 \%)$ only measured the impact of their intervention on soft drinks consumption $^{(38,73,76,92,101-103,104,106)}$. Slightly more than a third $(36 \cdot 1 \%)$ of studies included two types of SSB ( $k$ 13), generally soft drinks and fruit drinks ${ }^{(78-80,82,84,86,87,89-91,93-95)}$. Three studies (8.3\%) included three types of $\operatorname{SSB}^{(74,75,99)}$ and six studies $(16 \cdot 7 \%)$ reported information on four categories of $\operatorname{SSB}^{(81,83,85,96,98,107)}$. Finally, three studies $(8 \cdot 3 \%)$ included five types of $\mathrm{SSB}^{(77,88,97)}$ and among them, only one study ${ }^{(88)}$ included all of our five categories of SSB, namely soft drinks, fruit drinks, sports drinks, energy drinks and sweetened tea or coffee (iced or hot) ${ }^{(70)}$.

\section{Study designs and quality of studies}

Thirteen interventions (36.1\%) were RCT or cluster $\operatorname{RCT}^{(76,77,81,83,85,87,91,99,100,102,104,105,107)}$. A third of studies $(33 \cdot 3 \%$, $k$ 12) adopted a one-group pre-post study design, mainly those aimed at evaluating the impact of nutrition policies in schools (i.e. SSB consumption pre- and postpolicy) ${ }^{(38,73,78,80,84,88,89,93,95,97,98,106)}$. Eleven interventions (30.6\%) used quasi-experimental designs ${ }^{(74,75,79,82,86,90,92,94,96,101,103)}$.

Over $60 \%(61 \cdot 1 \%)$ of studies $(k 22)$ received a weak global rating for their quality according to the EPHPP tool ${ }^{(38,74-76,78,83,85,86,88-94,96,97,100,101,103,104,107)}$. Over $80 \%$ $(81 \cdot 8 \%)$ of quasi-experimental studies $(k 9)$ received a weak global rating ${ }^{(74,75,86,90,92,94,96,101,103)}$, followed by one-group pre-post studies $(50 \cdot 0 \%, k \quad 6)^{(38,78,88,89,93,97)}$ and RCT $(53 \cdot 8 \%, k 7)^{(76,83,85,91,100,104,107)}$. The three most frequent reasons for a weak global rating were: (i) presence of a selection bias; (ii) no blinding; and (iii) the data collection tool was not valid or reliable. A third (33.3\%) of studies $(k 12)$ received a global rating of moderate quality $^{(73,77,79,81,82,84,87,95,98,99,105,106)}$. Close to $40 \%(38 \cdot 5 \%)$ of RCT $(k, 5)$ received a global moderate rating ${ }^{(77,81,87,99,105)}$, followed by one-group pre-post studies $(33.3 \%$,

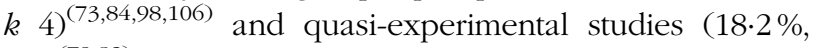
$k 2)^{(79,82)}$. Finally, only two studies received a strong global rating according to the EPHPP tool. One was a onegroup pre-post study that evaluated the impact of a school nutrition policy on SBB consumption using validated food records ${ }^{(80)}$. The other was an RCT on healthy eating and physical activity which used a validated self-administered FFQ to assess SSB consumption ${ }^{(102)}$.

\section{Results of interventions}

Over $70 \%(72 \cdot 2 \%, k 26)$ of all interventions, regardless of whether they targeted individuals, their environment or both, were effective in decreasing SSB consumption ${ }^{(38,73-77,79-82,84,88-93,95,96,98-100,104-107)}$. Their efficacy, however, varied according to the type of intervention (see Table 2). The ten legislative/environmental studies had the highest success rate, with nine studies (90.0\%) reporting a significant reduction in SSB consumption $^{(73,74,79,80,82,84,93,95,98)}$ and only one study with no significant reduction in SSB consumption following changes in the school food environment ${ }^{(94)}$. The twenty educational/behavioural interventions and the six interventions that were both educational/behavioural and legislative/environmental were almost equally effective in reducing SSB consumption, with success rates of $65 \cdot 0 \%\left(\begin{array}{ll}k & 13\end{array}\right)^{(38,76,77,81,88,90-92,96,99,100,104,105)}$ and $66 \cdot 7 \%$ ( $k$ 4 $)^{(75,89,106,107)}$, respectively. It is noteworthy that one legislative/environmental study ${ }^{(98)}$ and one educational/ behavioural intervention ${ }^{(38)}$ reported significant increases in SSB consumption post-intervention.

\section{Theory used in designing interventions}

Among the twenty-six interventions that had an educational/behavioural component, over $60 \%(61.5 \%$, $k$ 16) of studies were based on behavioural theories ${ }^{(75-78,83,85,87,91,92,96,97,99-102,107)}$. The theory most frequently used to design this type of intervention was the $\mathrm{SCT}^{(46)}$ and its predecessor the $\mathrm{SLT}^{(47)}$ $\left(\begin{array}{ll}k & 10\end{array}\right)^{(75-78,85,86,91,92,99,100)}$, followed by the $\mathrm{TPB}^{(48)}$ $\left(\begin{array}{ll}k & 4\end{array}\right)^{(75,76,102,107)}$, the $\operatorname{TTM}^{(49)}\left(\begin{array}{ll}k & 4\end{array}\right)^{(76,83,85,102)}$ and the $\operatorname{SDT}^{(50)}(k 3)^{(77,97,100)}$. It is worth noting that all of them are psychosocial theories (i.e. theories originating from social psychology) related to human motivation/intention. 
Other theories were mentioned by only one study. More than half $(56.3 \%, k 9)$ of theory-based interventions were effective in reducing SSB consumption $^{(75-77,91,92,96,99,100,107)}$.

\section{Behaviour change techniques used in interventions}

As previously mentioned, the majority of interventions targeted the reduction of SSB consumption as part of a general objective to promote healthy eating and physical activity or healthy eating alone. Consequently, the majority of the behaviour change techniques were directed towards promoting the larger behaviours (healthy eating and/or physical activity). Nevertheless, an effort was made to code only the behaviour change techniques related to SSB consumption and not those related to healthy eating (e.g. fruit and vegetable consumption) and/or physical activity when possible. When this was not possible, only the behaviour change techniques related to healthy eating and not those related to physical activity were coded. However, in some studies, the curriculum of the intervention that specifically targeted SSB and/or healthy eating was not stated.

The behaviour change techniques that were the most frequently used in interventions were providing information about the health consequences of performing the behaviour $(72 \cdot 2 \%, k 26)^{(38,75-78,82,83,85-92,96,97,99-107)}$, followed by restructuring the physical environment $(47 \cdot 2 \%, k 17)^{(73-75,79,80,82,84,86,89,93-95,98,102,105-107)}$, behavioural goal setting $(36 \cdot 1 \%, \quad k \quad 13)^{(38,76-78,81,83,85,87,91,92,97,100,107)}$, self-monitoring of behaviour $(33.3 \%, \quad k \quad 12)^{(38,76,77 \text {, }}$ $81,87,91,92,97,99,100,104,107)$, threat to health $(30 \cdot 6 \%$, k 11) $)^{(38,76-78,83,89,89,100,103-105)}$ and providing general social support $(30 \cdot 6 \%, k 11)^{(38,76,78,83,85,89,91,99,100,102,107)}$. The other behaviour change techniques were mentioned in ten or fewer different studies. The majority of legislative/ environmental studies used only one behaviour change technique (i.e. restructuring the physical environment) while the majority of interventions with an educational/ behavioural component used multiple behaviour change techniques. Restructuring the physical environment was a frequently used behaviour change technique given that all legislative/environmental studies aimed at evaluating the impact of school nutrition policies implied some changes in the school environment, such as banning SBB or replacing SSB by healthier alternatives (e.g. water, milk, $100 \%$ pure fruit juices). The majority of educational/ behavioural interventions explained to adolescents the negative health consequences of consuming SSB and they also sometimes included a component about threat to health when they further explained how chronic diseases related to SSB consumption, such as obesity and type 2 diabetes, can be detrimental to health. Behavioural goal setting (e.g. setting an objective to decrease one's own SSB consumption by one serving per day by next week), self-monitoring of behaviour (e.g. recording one's own daily consumption of SSB) and providing general social support were other behaviour change techniques commonly part of interventions with an educational/behavioural component. Parents $(72.7 \%, \quad k \quad 8)^{(38,76,78 \text {, }}$ $85,89,99,100,102)$ and/or friends $(45 \cdot 5 \%, k 5)^{(76,78,83,85,107)}$ were enlisted for social support and one study did not report from which specific persons social support was sought ${ }^{(91)}$. In some studies, parents received written material (newsletters, text messages, emails, postcards $)^{(38,85,99,100,102)}$ and/or were invited to school meetings ${ }^{(89,102)}$ to encourage them to support their adolescent to change his/her behaviour.

Finally, only four studies (11.1\%) used a control group which received some kind of intervention (i.e. active control group $)^{(81,87,91,96)}$. Among those studies, two had only one or two behaviour change techniques differentiating the experimental and the control group ${ }^{(91,96)}$ while the other two studies had six behaviour change techniques differentiating both groups ${ }^{(81,87)}$. Unfortunately, it was not possible to identify the most effective behaviour change technique given that studies with an educational/behavioural component often used a combination of different behaviour change techniques in their experimental group.

\section{Discussion}

The results of the present systematic review indicate that the majority of school-based interventions are effective at reducing SSB consumption among adolescents, although the overall rating of the interventions was frequently weak. This suggests that the school setting might represent a promising place to easily reach adolescents, regardless of their age, SES, cultural background and ethnicity $^{(32,37,38)}$. For example, in the present systematic review, a few studies targeted adolescents whose parents had a low $\operatorname{SES}^{(77,82,83,85,86,88,93,94,99-101)}$ or specific ethnic minorities in the $\mathrm{USA}^{(81,89,90)}$. Given that parents' lower SES can be associated with SSB consumption among young children ${ }^{(108)}$, schools - especially those in lowincome neighbourhoods - could be a good place to reach adolescents at high risk for SSB consumption.

According to the present findings, legislative/environmental interventions were the most effective while educational/behavioural interventions and those targeting both individuals and their environment were less, but both equally effective, at decreasing SSB consumption among adolescents. Overall, this suggests that governmental efforts to reduce availability and/or eliminate SSB in schools should be pursued. However, governmental nutrition policies can also have unintended consequences. For example, one study conducted in Canada reported that while frequency of SSB consumption decreased following a ban on SSB in schools, the volume of SBB (in millilitres) consumed increased ${ }^{(98)}$. In other words, adolescents might report consuming SSB less frequently 
simply because they drink larger quantities each time they consume SSB. This could reflect a trend of the industry to continuously increase the size of the SBB it sells over the years ${ }^{(109)}$. Another study in the USA observed that while the overall mean servings of SSB in school decreased following the implementation of a school nutrition policy, three times more adolescents mentioned bringing SBB from home post-policy ${ }^{(80)}$. Similarly, the results of another study not included in the present review (the outcome was SSB sales) suggested that when there is a ban on SSB in schools, some adolescents instead buy SSB in stores located on their school commute ${ }^{(110)}$. One way of possibly avoiding these unintended consequences could be to provide educational/behavioural activities among adolescents and their parents about the negative consequences associated with consuming SSB as well as tips to promote drinking heathier alternatives and to overcome the barriers that could be encountered. This could help adolescents make healthy choices when they are outside school and parents could also support them by providing non-SSB at home, such as water and milk. In fact, substituting SBB with water and milk can have a positive effect on body fatness of adolescents ${ }^{(111)}$. Yet, in the present review, only six interventions targeted both individuals and their environment as recommended by ecological models often used to design public health interventions ${ }^{(39)}$ and by research specifically targeting obesity prevention and healthy eating among children and adolescents ${ }^{(69,112,113)}$.

Among studies including an educational/behavioural component, more than half were based on a psychosocial theory, such as the SCT/SLT, TPB, TTM and SDT, which are some of the most commonly used theories for developing public health interventions ${ }^{(114)}$. This is an interesting finding as over the years, a number of authors have advocated for the use of theory in designing public health interventions $^{(40-44,114,115)}$. In fact, among the theory-based interventions included in the present review, more than half of them were effective in reducing SSB consumption among adolescents. While at first sight this might seem like a rather low success rate, current evidence regarding the efficacy of theory-based interventions is conflicting, with some studies reporting that theory-based interventions are more effective than those not theory-based ${ }^{(114,116)}$ while others report that both theory-based and non-theorybased interventions are equally effective in changing health behaviour ${ }^{(117)}$. Nevertheless, one advantage of using a theory is the potential to guide the choice of behaviour change techniques to use in interventions ${ }^{(51,52)}$.

It is interesting to note that while a majority of interventions report being based on the SCT/SLT, TPB, TTM and SDT, none of the most popular behaviour change techniques previously discussed - except providing information about health consequences, which is part of the SCT/SLT and the TPB - are recommended by any of these psychosocial theories ${ }^{(52)}$. Unfortunately, it is rather common that behaviour change techniques used in interventions are not necessarily related to the theory that interventions are supposed to be based on ${ }^{(117)}$, which is why some authors came up with the expression 'theoryinspired' instead of 'theory-based' to describe certain interventions ${ }^{(54)}$. This could also explain the somewhat low success rate of theory-based interventions because using behaviour change techniques linked to the chosen theory should be more effective at changing behaviour than using theory-irrelevant behaviour change techniques $^{(117)}$.

Finally, in the present review, providing information on the health consequences related to SSB consumption was the most frequently used behaviour change technique. While knowledge of the health benefits and risks of a particular behaviour is a requirement and one of the first steps for behaviour change, it is usually deemed not sufficient to engender behaviour change according to the author of the SCT/SLT ${ }^{(118)}$. Other behaviour change techniques need to be used in conjunction with this strategy. Self-monitoring of behaviour was another behaviour change technique often used in interventions aimed at decreasing SSB consumption among adolescents. In fact, according to previous reviews, self-monitoring is one of the most commonly used techniques to promote physical activity among overweight/obese adults ${ }^{(119)}$ and it is also consistently associated with behaviour change ${ }^{(120,121)}$ and with weight $\operatorname{loss}^{(122)}$, which might explain its popularity. Behavioural goal setting was also a prevalent behaviour change technique to encourage adolescents to reduce their SSB consumption. Previous reviews found that goal setting is an effective strategy to promote health behaviour changes among overweight/ obese adults ${ }^{(123)}$ and people with type 2 diabetes $^{(124)}$. Providing general social support was another frequent component of interventions whose objective was to lower SSB consumption among adolescents. As previously mentioned, parents play an important role in encouraging their adolescents to develop healthy habits outside school. In the articles included in the present systematic review, they were the persons most frequently solicited for social support and some studies even targeted them in their interventions by sending them written material and/or inviting them to school meetings. In fact, according to ecological models used in public health ${ }^{(39)}$ and supported by empirical work aimed at improving nutrition and preventing obesity among youth ${ }^{(32,69,125)}$, interventions that target different levels of social influences, such as adolescents, their parents and the school environment, should be more effective at changing health behaviours than those simply aimed at individuals.

\section{Recommendations for future studies}

More studies targeting individuals and their environment, as recommended by ecological models used in public health ${ }^{(39)}$, are needed to avoid unintended consequences 
associated with interventions only aimed at changing the school food environment. Ideally, theory-based interventions should choose behaviour change techniques relevant to their choice of theory. Authors whose interventions are aimed at multiple health behaviours, such as healthy eating (including SSB consumption) and physical activity, should clearly report which behaviour change techniques were used for each behaviour. This information would help distinguish which behaviour change techniques are the most effective for improving each specific behaviour. To facilitate replication, authors are encouraged to briefly explain how each behaviour change technique was used. In many of the included studies, authors simply reported using goal setting and self-monitoring without specifying how this was applied. This information is important given that there is evidence that increasing the level of specificity of certain behaviour change techniques increases the chances of successfully changing behaviour. For example, action planning is a behaviour change technique similar to goal setting, but that requires more detailed planning, such as specifying at least one of the following components: the context, frequency, intensity and duration of the behaviour ${ }^{(53)}$. Recent studies found that adults who made more specific action plans had greater odds of attaining their goal concerning fruit or vegetable intake or physical activity $^{(126)}$ and experienced greater weight loss when they had high weight-loss goals ${ }^{(127)}$. To improve the quality of their study and also facilitate comparison across studies, authors are also advised to use a valid and reliable measure of SSB consumption to obtain precise information on both frequency (e.g. times per day or per week) and quantity (e.g. in millilitres or fluid ounces) of SBB consumed. Finally, authors are encouraged to not just include soft drinks in their definition of SSB given that other types of SSB such as sports drinks and energy drinks are increasingly more popular among adolescents ${ }^{(128)}$ and are equally detrimental to health ${ }^{(55,129-131)}$. There is also evidence that when only sodas are banned in schools, adolescents replace them by other SBB, such as sports drinks, energy drinks and sweetened coffee and tea ${ }^{(132)}$. However, when including different types of SSB, authors are advised to report on types of SSB separately in case their intervention has a different effect on each drink included in their definition.

\section{Limitations of the systematic review}

Unfortunately, it was not possible to conduct a metaanalysis on the efficacy of interventions given the important heterogeneity observed between studies. Part of this heterogeneity could result from the inclusion of different individual measures of SSB consumption (e.g. millilitres or number of glasses per day or per week, percentage of individuals who reported consuming a given quantity of SSB, etc.) and also different measures of this outcome (e.g. different types of SSB reported separately or collectively) in the present systematic review. This decision was made since there was no consensus on how to measure individual SSB consumption. In fact, a recent review of methods to assess intake of SSB among adults, adolescents and children concluded that there is a need for an agreed definition of SSB among instruments measuring this behaviour ${ }^{(62)}$. At the same time, a strength of the present review was the inclusion of different types of intervention, such as educational/behavioural and legislative/environmental interventions, as both can inform the development of school-based interventions and are relevant for public health. The inclusion of different study designs was another strength ${ }^{(133)}$, since including only RCT would have excluded studies reporting the efficacy of school nutrition policies. It was also not possible to verify the presence of a publication bias, which could explain why the majority of the studies reported a significant reduction of SSB consumption after their intervention. To lower the risk of encountering this bias, grey literature (i.e. unpublished trials) was included in the present review.

\section{Conclusions}

To our knowledge, the present study is the first to systematically review school-based interventions aimed at reducing SSB consumption among adolescents. It also applied the taxonomy of Cane et al. ${ }^{(53)}$ to classify the behaviour change techniques used in interventions. Another novel aspect of the current review is the assessment of the quality of each study using the EPHPP tool ${ }^{(71)}$. As such, the present review contributes to identify gaps in knowledge and suggest new directions for people wishing to develop school-based interventions to effectively reduce SSB consumption among adolescents.

School-based interventions show promising results to reduce SSB consumption among adolescents and governmental efforts to reduce availability and/or eliminate SSB in schools should be pursued. More studies targeting individuals and their environment, as recommended by ecological models used in public health ${ }^{(39)}$, are needed to avoid unintended consequences associated with interventions aimed only at changing the school food environment. Finally, it is hoped that these findings and the growing rates of obesity among adolescents will encourage public health authorities and researchers to pursue their efforts to encourage adolescents to adopt healthy drinking habits, such as replacing SSB by water or milk ${ }^{(111)}$, which could be maintained throughout life.

\section{Acknowledgements}

Acknowledgements: The authors would like to acknowledge the contribution of Marie-Ève Émond-Beaulieu who helped with the search strategy. Financial support: This 
work was supported by a grant from Université du Québec à Rimouski (UQAR) (grant number TRF C3-751325). UQAR had no role in the design, analysis or writing of this article. Conflict of interest: None. Authorship: L.-A.V.-I. conducted the search strategy and its update in each database, selected studies for their inclusion in the systematic review, extracted and analysed the data, and wrote the first draft of the manuscript with Do.B. Do.B. designed the review with L.-A.V.-I., registered the protocol in PROSPERO, confirmed the study selection, did the second data extraction for a selection of articles and critically revised the manuscript. A.B.-G., D.a.B. and M.D. did the second data extraction for a selection of articles and critically revised the paper. C.S. and V.P. critically revised the manuscript. Ethics of human subject participation: Not applicable.

\section{Supplementary material}

To view supplementary material for this article, please visit https://doi.org/10.1017/S1368980017000076

\section{References}

1. World Health Organization (2003) Diet, Nutrition and the Prevention of Chronic Diseases. Joint WHO/FAO Expert Consultation. WHO Technical Report Series no. 916. Geneva: WHO.

2. Ahluwalia N, Dalmasso P, Rasmussen M et al. (2015) Trends in overweight prevalence among 11-, 13- and 15 -year-olds in 25 countries in Europe, Canada and USA from 2002 to 2010. Eur J Public Health 25, Suppl. 2, 28-32.

3. Ogden CL, Carroll MD, Lawman HG et al. (2016) Trends in obesity prevalence among children and adolescents in the United States, 1988-1994 through 2013-2014. JAMA 315, 2292-2299.

4. Roberts KC, Shields M, de Groh M et al. (2012) Overweight and obesity in children and adolescents: results from the 2009 to 2011 Canadian Health Measures Survey. Health Rep 23, 3-7.

5. World Health Organization (2000) Obesity: Preventing and Managing the Global Epidemic. Report of a WHO Consultation. WHO Technical Report Series no. 894. Geneva: WHO.

6. World Health Organization (2015) Guideline: Sugars Intake for Adults and Children. Geneva: WHO.

7. Yang Q, Zhang Z, Gregg EW et al. (2014) Added sugar intake and cardiovascular diseases mortality among US adults. JAMA Intern Med 174, 516-524.

8. Johnson RK, Appel LJ, Brands M et al. (2009) Dietary sugars intake and cardiovascular health: a scientific statement from the American Heart Association. Circulation 120, 1011-1020.

9. Liu S, Willett WC, Stampfer MJ et al. (2000) A prospective study of dietary glycemic load, carbohydrate intake, and risk of coronary heart disease in US women. Am J Clin Nutr 71, 1455-1461.

10. Malik VS, Popkin BM, Bray GA et al. (2010) Sugarsweetened beverages, obesity, type 2 diabetes mellitus, and cardiovascular disease risk. Circulation 121, 1356-1364.
11. Bray GA, Nielsen SJ \& Popkin BM (2004) Consumption of high-fructose corn syrup in beverages may play a role in the epidemic of obesity. Am J Clin Nutr 79, 537-543.

12. Pollock NK, Bundy V, Kanto W et al. (2012) Greater fructose consumption is associated with cardiometabolic risk markers and visceral adiposity in adolescents. $J$ Nutr 142, 251-257.

13. Sievenpiper JL, de Souza RJ, Mirrahimi A et al. (2012) Effect of fructose on body weight in controlled feeding trials: a systematic review and meta-analysis. Ann Intern Med 156, 291-304.

14. Te Morenga L, Mallard S \& Mann J (2013) Dietary sugars and body weight: systematic review and meta-analyses of randomised controlled trials and cohort studies. BMJ $\mathbf{3 4 6}$, e7492.

15. Wang H, Steffen LM, Zhou X et al. (2013) Consistency between increasing trends in added-sugar intake and body mass index among adults: the Minnesota Heart Survey, 1980-1982 to 2007-2009. Am J Public Health 103, 501-507.

16. Olsen NJ \& Heitmann BL (2009) Intake of calorically sweetened beverages and obesity. Obes Rev 10, 68-75.

17. Cozma AI, Sievenpiper JL, de Souza RJ et al. (2012) Effect of fructose on glycemic control in diabetes: a systematic review and meta-analysis of controlled feeding trials. Diabetes Care 35, 1611-1620.

18. Basu S, Yoffe P, Hills N et al. (2013) The relationship of sugar to population-level diabetes prevalence: an econometric analysis of repeated cross-sectional data. PLoS One 8, e 57873

19. Davis JN, Ventura EE, Weigensberg MJ et al. (2005) The relation of sugar intake to $\beta$ cell function in overweight Latino children. Am J Clin Nutr 82, 1004-1010.

20. Malik VS, Popkin BM, Bray GA et al. (2010) Sugarsweetened beverages, obesity, type 2 diabetes melitus and cardiovascular disease risk. Circulation 121, 1356-1364.

21. Sievenpiper JL, Carleton AJ, Chatha S et al. (2009) Heterogeneous effects of fructose on blood lipids in individuals with type 2 diabetes: systematic review and meta-analysis of experimental trials in humans. Diabetes Care 32, 1930-1937.

22. Welsh JA, Sharma A, Abramson JL et al. (2010) Caloric sweetener consumption and dyslipidemia among US adults. JAMA 303, 1490-1497.

23. Larsson SC, Bergkvist L \& Wolk A (2006) Consumption of sugar and sugar-sweetened foods and the risk of pancreatic cancer in a prospective study. Am J Clin Nutr 84, $1171-1176$

24. Moynihan PJ \& Kelly SAM (2014) Effect on caries of restricting sugars intake: systematic review to inform WHO guidelines. J Dent Res $\mathbf{9 3}, 8-18$.

25. Ordre professionnel des diététistes du Québec (2013) Boissons gazeuses: rafraichissantes... mais à quel prix? http://publications.msss.gouv.qc.ca/msss/fichiers/2010/10289-16F.pdf (accessed April 2015).

26. Ng SW, Ni Mhurchu C, Jebb SA et al. (2012) Patterns and trends of beverage consumption among children and adults in Great Britain, 1986-2009. Br J Nutr 108, 536-551.

27. Sanchez-Pimienta TG, Batis C, Lutter CK et al. (2016) Sugar-sweetened beverages are the main sources of added sugar intake in the Mexican population. $J$ Nutr 146, issue 9, 1888S-1896S.

28. Popkin BM (2010) Patterns of beverage use across the lifecycle. Physiol Behav 100, 4-9.

29. Garriguet D (2008) Consommation de boissons par les enfants et les adolescents. Rapports sur la Santé 19, 17-22.

30. Breinbauer C \& Maddaleno M (2005) Youth: Choices and Change. Promoting Healthy Behaviors in Adolescents. PAHO Scientific and Technical Publication no. 594. Washington, DC: Pan American Health Organization. 
31. Baril G, Paquette M-C \& Ouimet A-M (2014) Ados 12-14: les dimensions socioculturelles des pratiques alimentaires et d'activité physique des adolescents. Québec, QC: Institut national de santé publique du Québec, Gouvernement du Québec.

32. Flynn MAT, McNeil DA, Maloff B et al. (2006) Reducing obesity and related chronic disease risk in children and youth: a synthesis of evidence with 'best practice' recommendations. Obes Rev 7, 7-66.

33. Ministère de l'Éducation, du Loisir et du Sport (2007) Pour un virage santé à l'école. Politique-cadre pour une saine alimentation et un mode de vie physiquement actif. Québec, QC: MELS, Gouvernement du Québec.

34. Story M, Neumark-Sztainer D \& French S (2002) Individual and environmental influences on adolescent eating behaviors. J Am Diet Assoc 102, 3 Suppl., S40-S51.

35. Taylor JP, Evers S \& McKenna M (2005) Determinants of healthy eating in children and youth. Can J Public Health 96, Suppl. 3, S20-S26.

36. Casey L \& Crumley E (2004) Addressing Childhood Obesity: The Evidence for Action. Ottawa, ON: Canadian Association of Paediatric Health Centres, Paediatric Chairs of Canada, CIHR Institute of Nutrition, Metabolism and Diabetes.

37. Bandura A (1997) Exercise of personal and collective efficacy in changing society. In Self-Efficacy in Changing Societies, pp. 1-45 [A Bandura, editor]. Cambridge: Cambridge University Press.

38. Jones M, Lynch KT, Kass AE et al. (2014) Healthy weight regulation and eating disorder prevention in high school students: a universal and targeted web-based intervention. $J$ Med Internet Res 16, e57.

39. Sallis JF, Owen N \& Fisher EB (2008) Ecological models of health behavior. In Health Behavior and Health Education: Theory, Research, and Practice, pp. 465-485 [K Glanz, BK Rimer and K Viswanath, editors]. San Francisco, CA: Jossey-Bass.

40. Baranowski T, Lin LS, Wetter DW et al. (1997) Theory as mediating variables: why aren't community interventions working as desired? Ann Epidemiol 7, Suppl., S89-S95.

41. Brug J, Oenema A \& Ferreira I (2005) Theory, evidence and intervention mapping to improve behavior nutrition and physical activity interventions. Int J Behav Nutr Phys Act 2, 2.

42. Fishbein M \& Yzer MC (2003) Using theory to design effective health behavior interventions. Commun Theory 13, 164-183.

43. King AC (2015) Theory's role in shaping behavioral health research for population health. Int J Behav Nutr Phys Act 12, 146

44. Michie S \& Abraham C (2004) Interventions to change health behaviours: evidence-based or evidence-inspired? Psychol Health 19, 29-49.

45. Rothman AJ (2004) 'Is there nothing more practical than a good theory?': why innovations and advances in health behavior change will arise if interventions are used to test and refine theory. Int J Behav Nutr Phys Act 1, 11.

46. Bandura A (1977) Self-efficacy: toward a unifying theory of behavioral change. Psychol Rev 84, 191-215.

47. Bandura A (1977) Social Learning Theory. Englewood Cliffs, NJ: Prentice-Hall.

48. Ajzen I (1991) The theory of planned behavior. Organ Behav Hum Decis Process 50, 179-211.

49. Prochaska JO \& DiClemente CC (1983) Stages and processes of self-change of smoking: toward an integrative model of change. J Consult Clin Psychol 51, 390-365.

50. Ryan RM \& Deci EL (2000) Intrinsic and extrinsic motivations: classic definitions and new directions. Contemp Educ Psychol 25, 54-67.
51. Michie S, Johnston M, Francis J et al. (2008) From theory to intervention: mapping theoretically derived behavioural determinants to behaviour change techniques. Appl Psychol Int Rev 57, 660-680.

52. Abraham C \& Michie S (2008) A taxonomy of behavior change techniques used in interventions. Health Psychol 27, 379-387.

53. Cane J, Richardson M, Johnston M et al. (2015) From lists of behaviour change techniques (BCTs) to structured hierarchies: comparison of two methods of developing a hierarchy of BCTs. Br J Health Psychol 20, 130-150.

54. Michie S, Carey RN, Johnston M et al. (2016) From theoryinspired to theory-based interventions: a protocol for developing and testing a methodology for linking behaviour change techniques to theoretical mechanisms of action. Ann Behav Med (Epublication ahead of print version).

55. Ali F, Rehman H, Babayan Z et al. (2015) Energy drinks and their adverse health effects: a systematic review of the current evidence. Postgrad Med 127, 308-322.

56. Forshee RA, Anderson PA \& Storey ML (2008) Sugarsweetened beverages and body mass index in children and adolescents: a meta-analysis. Am J Clin Nutr 87, $1662-1671$.

57. Malik VS, Pan A, Willett WC et al. (2013) Sugar-sweetened beverages and weight gain in children and adults: a systematic review and meta-analysis. Am J Clin Nutr 98, $1084-1102$.

58. Trumbo PR \& Rivers CR (2014) Systematic review of the evidence for an association between sugar-sweetened beverage consumption and risk of obesity. Nutr Rev $\mathbf{7 2}$, 566-574

59. Keller A \& Bucher Della Torre S (2015) Sugar-sweetened beverages and obesity among children and adolescents: a review of systematic literature reviews. Child Obes 11, 338-346.

60. Bucher Della Torre S, Keller A, Laure Depeyre J et al. (2016) Sugar-sweetened beverages and obesity risk in children and adolescents: a systematic analysis on how methodological quality may influence conclusions. I Acad Nutr Diet 116, 638-659.

61. Weed DL, Althuis MD \& Mink PJ (2011) Quality of reviews on sugar-sweetened beverages and health outcomes: a systematic review. Am J Clin Nutr 94, 1340-1347.

62. Riordan F, Ryan K, Perry IJ et al. (2016) A systematic review of methods to assess intake of sugar-sweetened beverages among healthy European adults and children: a DEDIPAC (DEterminants of DIet and Physical Activity) study. Public Health Nutr (Epublication ahead of print version).

63. Levy DT, Friend KB \& Wang YC (2011) A review of the literature on policies directed at the youth consumption of sugar sweetened beverages. Adv Nutr 2, issue 2, 182S-200S.

64. Powell LM, Chriqui JF, Khan T et al. (2013) Assessing the potential effectiveness of food and beverage taxes and subsidies for improving public health: a systematic review of prices, demand and body weight outcomes. Obes Rev 14, 110-128.

65. Cabrera Escobar MA, Veerman JL, Tollman SM et al. (2013) Evidence that a tax on sugar sweetened beverages reduces the obesity rate: a meta-analysis. BMC Public Health 13, 1072.

66. Avery A, Bostock L \& McCullough F (2015) A systematic review investigating interventions that can help reduce consumption of sugar-sweetened beverages in children leading to changes in body fatness. J Hum Nutr Diet 28, 52-64.

67. Lane H, Porter K, Estabrooks P et al. (2016) A systematic review to assess sugar-sweetened beverage interventions 
for children and adolescents across the socioecological model. J Acad Nutr Diet 116, 1295-1307.e6.

68. Tipton JA (2015) Reducing sugar-sweetened beverage intake among students: school-based programs and policies that work. NASN Sch Nurse 31, 102-110.

69. Sharma M (2006) School-based interventions for childhood and adolescent obesity. Obes Rev 7, 261-269.

70. Centers for Disease Control and Prevention (2010) The CDC Guide to Strategies for Reducing the Consumption of Sugar-Sweetened Beverages. Atlanta, GA: CDC.

71. Armijo-Olivo S, Stiles CR, Hagen NA et al. (2012) Assessment of study quality for systematic reviews: a comparison of the Cochrane Collaboration Risk of Bias Tool and the Effective Public Health Practice Project Quality Assessment Tool: methodological research. J Eval Clin Pract 18, $12-18$

72. Higgins JPT \& Green S (2011) Cochrane Handbook for Systematic Reviews of Interventions Version 5.0.2. http:// www.cochrane-handbook.org (accessed March 2011).

73. Bauhoff S (2014) The effect of school district nutrition policies on dietary intake and overweight: a synthetic control approach. Econ Hum Biol 12, 45-55.

74. Blum JE, Davee AM, Beaudoin CM et al. (2008) Reduced availability of sugar-sweetened beverages and diet soda has a limited impact on beverage consumption patterns in Maine high school youth. J Nutr Educ Behav 40, 341-347.

75. Bogart LM, Elliott MN, Uyeda K et al. (2011) Preliminary healthy eating outcomes of $\mathrm{SNaX}$, a pilot communitybased intervention for adolescents. $J$ Adolesc Health $\mathbf{4 8}$, 196-202.

76. Casazza K (2006) A computer based approach to improve the dietary and physical activity patterns of a diverse group of adolescents. PhD Thesis, Florida International University.

77. Contento IR, Koch PA, Lee H et al. (2010) Adolescents demonstrate improvement in obesity risk behaviors after completion of choice, control \& change, a curriculum addressing personal agency and autonomous motivation. J Am Diet Assoc 110, 1830-1839.

78. Cordeira KL (2012) Effects of a health advocacy program to promote physical activity and nutrition behaviors among adolescents: an evaluation of the Young Leaders for Healthy Change Program. MPH Disseration, University of Texas School of Public Health.

79. Cradock AL, McHugh A, Mont-Ferguson H et al. (2011) Effect of school district policy change on consumption of sugar-sweetened beverages among high school students, Boston, Massachusetts, 2004-2006. Prev Chronic Dis 8, A74.

80. Cullen KW, Watson K \& Zakeri I (2008) Improvements in middle school student dietary intake after implementation of the Texas Public School Nutrition Policy. Am J Public Health 98, 111-117.

81. Davis JN, Ventura EE, Alexander KE et al. (2007) Feasibility of a home-based versus classroom-based nutrition intervention to reduce obesity and type 2 diabetes in Latino youth. Int J Pediatr Obes 2, 22-30.

82. Greece JA (2011) Behavioral impact of a school-based healthy eating intervention for at-risk children. ScD Dissertation, Boston University.

83. Lao LS (2011) Evaluation of a social networking based SNAP-Ed nutrition curriculum on behavior change. MS Disseration, University of Rhode Island.

84. Nanney MS, Maclehose R, Kubik MY et al. (2014) Recommended school policies are associated with student sugary drink and fruit and vegetable intake. Prev Med $\mathbf{6 2}$, 179-181.

85. Neumark-Sztainer DR, Friend SE, Flattum CF et al. (2010) New moves-preventing weight-related problems in adolescent girls a group-randomized study. Am J Prev Med 39, 421-432.
86. Patel AI, Bogart LM, Elliott MN et al. (2011) Increasing the availability and consumption of drinking water in middle schools: a pilot study. Prev Chronic Dis $\mathbf{8}, \mathrm{A} 60$.

87. Pbert L, Druker S, Gapinski MA et al. (2013) A school nurse-delivered intervention for overweight and obese adolescents. J Sch Health 83, 182-193.

88. Smith LH \& Holloman C (2014) Piloting 'sodabriety': a school-based intervention to impact sugar-sweetened beverage consumption in rural Appalachian high schools. J Sch Health 84, 177-184.

89. Teufel NI \& Ritenbaugh CK (1998) Development of a primary prevention program: insight gained in the Zuni Diabetes Prevention Program. Clin Pediatr 37, 131-141.

90. Thiele MC \& Boushey CJ (1989) Soft drink consumption among Yup'ik Eskimo teenagers. Alaska Med 31, 1-3.

91. Whittemore R, Jeon S \& Grey M (2013) An internet obesity prevention program for adolescents. J Adolesc Health 52, 439-447.

92. Winett RA, Roodman AA, Winett SG et al. (1999) The effects of the Eat4Life Internet-based health behavior program on the nutrition and activity practices of high school girls. J Gender Culture Health 4, 239-254.

93. Woodward-Lopez G, Gosliner W, Samuels SE et al. (2010) Lessons learned from evaluations of California's statewide school nutrition standards. Am J Public Health 100 , 2137-2145.

94. Wordell D, Daratha K, Mandal B et al. (2012) Changes in a middle school food environment affect food behavior and food choices. J Acad Nutr Diet 112, 137-141.

95. Nanney MS, MacLehose RF, Kubik MY et al. (2016) School obesity prevention policies and practices in Minnesota and student outcomes: a longitudinal cohort study. Am J Prev Med 51, 656-663.

96. Lo E, Coles R, Humbert ML et al. (2008) Beverage intake improvement by high school students in Saskatchewan, Canada. Nutr Res 28, 144-150.

97. Malbon CA (2012) Examining the influence of an online health behaviour support tool for high school aged youth. MSc Dissertation, University of Victoria (Canada).

98. McGoldrick KA (2006) Sweetened beverage consumption of Peel adolescents: nutritional correlates and influence of the school and neighbourhood food environments, MSc Dissertation, University of Waterloo.

99. Collins CE, Dewar DL, Schumacher TL et al. (2014) 12 month changes in dietary intake of adolescent girls attending schools in low-income communities following the NEAT Girls cluster randomized controlled trial. Appetite 73, 147-155.

100. Smith JJ, Morgan PJ, Plotnikoff RC et al. (2014) Smartphone obesity prevention trial for adolescent boys in lowincome communities: the ATLAS RCT. Pediatrics 134, e723-e731.

101. Dubuy V, De Cocker K, De Bourdeaudhuij I et al. (2014) Evaluation of a real world intervention using professional football players to promote a healthy diet and physical activity in children and adolescents from a lower socio-economic background: a controlled pretestposttest design. BMC Public Health 14, 457.

102. Haerens L, Deforche B, Maes L et al. (2006) Evaluation of a 2-year physical activity and healthy eating intervention in middle school children. Health Educ Res 21, 911-921.

103. da Silva Vargas IC, Sichieri R, Sandre-Pereira G et al. (2011) Evaluation of an obesity prevention program in adolescents of public schools. Rev Saude Publica 45, 59-68.

104. Wing YK, Chan NY, Yu MWM et al. (2015) A school-based sleep education program for adolescents: a cluster randomized trial. Pediatrics 135, e635-e643.

105. Singhal N, Misra A, Shah P et al. (2010) Effects of controlled school-based multi-component model of nutrition and lifestyle interventions on behavior modification, 
anthropometry and metabolic risk profile of urban Asian Indian adolescents in North India. Eur J Clin Nutr 64, 364-373.

106. Bae SG, Kim JY, Kim KY et al. (2012) Changes in dietary behavior among adolescents and their association with government nutrition policies in Korea, 2005-2009. J Prev Med Public Health 45, 47-59.

107. Yildirim M, Singh AS, te Velde SJ et al. (2013) Mediators of longitudinal changes in measures of adiposity in teenagers using parallel process latent growth modeling. Obesity (Silver Spring) 21, 2387-2395.

108. Mazarello Paes V, Hesketh K, O'Malley C et al. (2015) Determinants of sugar-sweetened beverage consumption in young children: a systematic review. Obes Rev $\mathbf{1 6}$ 903-913.

109. Nielsen SJ \& Popkin BM (2004) Changes in beverage intake between 1977 and 2001. Am J Prev Med 27, 205-210.

110. Grummon AH, Oliva A, Hampton KE et al. (2015) Association between student purchases of beverages during the school commute and in-school consumption of sugar-sweetened beverages, San Francisco Bay area, 2013. Prev Chronic Dis 12, E220.

111. Zheng M, Rangan A, Olsen NJ et al. (2015) Substituting sugar-sweetened beverages with water or milk is inversely associated with body fatness development from childhood to adolescence. Nutrition 31, 38-44.

112. Veugelers PJ \& Fitzgerald AL (2005) Effectiveness of school programs in preventing childhood obesity: a multilevel comparison. Am J Public Health 95, 432-435.

113. Flodmark CE, Marcus C \& Britton M (2006) Interventions to prevent obesity in children and adolescents: a systematic literature review. Int J Obes (Lond) 30, 579-589.

114. Glanz K \& Bishop DB (2010) The role of behavioral science theory in development and implementation of public health interventions. Annu Rev Public Health 31, 399-418.

115. Bartholomew LK \& Mullen PD (2011) Five roles for using theory and evidence in the design and testing of behavior change interventions. J Public Health Dent 71, Suppl. 1, S20-S33.

116. Webb TL, Joseph J, Yardley L et al. (2010) Using the internet to promote health behavior change: a systematic review and meta-analysis of the impact of theoretical basis, use of behavior change techniques, and mode of delivery on efficacy. J Med Internet Res 12, e4.

117. Prestwich A, Sniehotta FF, Whittington C et al. (2014) Does theory influence the effectiveness of health behavior interventions? Meta-analysis. Health Psychol 33, 465-474.

118. Bandura A (2004) Health promotion by social cognitive means. Health Educ Behav 31, 143-164.

119. Bélanger-Gravel A, Godin G, Vézina-Im LA et al. (2011) The effect of theory-based interventions on physical activity participation among overweight/obese individuals: a systematic review. Obes Rev 12, 430-439.
120. Harkin B, Webb TL, Chang BPI et al. (2016) Does monitoring goal progress promote goal attainment? A metaanalysis of the experimental evidence. Psychol Bull 142, 198-229.

121. Michie S, Abraham C, Whittington C et al. (2009) Effective techniques in healthy eating and physical activity interventions: a meta-regression. Health Psychol 28, 690-701.

122. Burke LE, Wang J \& Sevick MA (2011) Self-monitoring in weight loss: a systematic review of the literature. J Am Diet Assoc 111, 92-102.

123. Pearson ES (2012) Goal setting as a health behavior change strategy in overweight and obese adults: a systematic literature review examining intervention components. Patient Educ Couns 87, 32-42.

124. Miller CK \& Bauman J (2014) Goal setting: an integral component of effective diabetes care. Curr Diab Rep 14, 509 .

125. Waters E, de Silva-Sanigorski A, Hall BJ et al. (2011) Interventions for preventing obesity in children. Cochrane Database Syst Rev 12, CD001871.

126. Plaete J, De Bourdeaudhuij I, Verloigne M et al. (2016) The use and evaluation of self-regulation techniques can predict health goal attainment in adults: an explorative study. PeerJ 4, e1666.

127. Dombrowski SU, Endevelt R, Steinberg DM et al. (2016) Do more specific plans help you lose weight? Examining the relationship between plan specificity, weight loss goals, and plan content in the context of a weight management programme. BrJ Health Psychol 21, 989-1005.

128. Harris JL \& Munsell CR (2015) Energy drinks and adolescents: what's the harm? Nutr Rev 73, 247-257.

129. Richards G \& Smith AP (2016) A review of energy drinks and mental health, with a focus on stress, anxiety, and depression. J Caffeine Res 6, 49-63.

130. Lippi G, Cervellin G \& Sanchis-Gomar F (2016) Energy drinks and myocardial ischemia: a review of case reports. Cardiovasc Toxicol 16, 207-212.

131. Committee on Nutrition \& the Council on Sports Medicine and Fitness (2011) Sports drinks and energy drinks for children and adolescents: are they appropriate? Pediatrics 127, 1182-1189.

132. Taber DR, Chriqui JF, Vuillaume R et al. (2015) The association between state bans on soda only and adolescent substitution with other sugar-sweetened beverages: a cross-sectional study. Int J Behav Nutr Phys Act 12, Suppl. 1, S7.

133. Valentine JC \& Thompson SG (2013) Issues relating to confounding and meta-analysis when including nonrandomized studies in systematic reviews on the effects of interventions. Res Synth Methods 4, 26-35.

134. Moher D, Liberati A, Tetzlaff J et al. (2009) Preferred reporting items for systematic reviews and metaanalyses: the PRISMA statement. Ann Intern Med 151, 264-269, W64. 